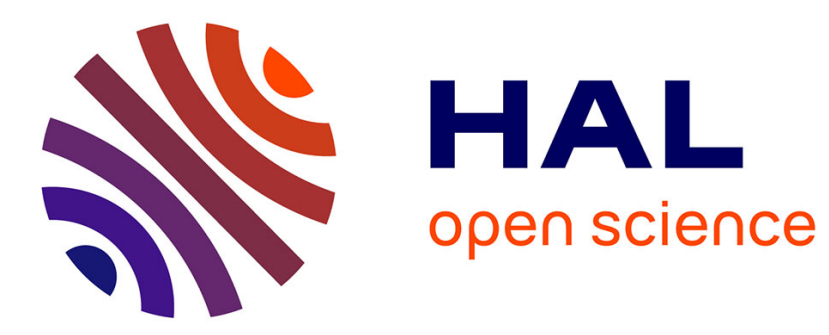

\title{
La prostitution en clubs dans les régions frontalières de la République tchèque
}

\author{
Mathilde Darley
}

\section{To cite this version:}

Mathilde Darley. La prostitution en clubs dans les régions frontalières de la République tchèque. Revue française de sociologie, 2007, 48 (2), pp.273-306. 10.3917/rfs.482.0273 . halshs-00648934

\section{HAL Id: halshs-00648934 \\ https://shs.hal.science/halshs-00648934}

Submitted on 30 Aug 2021

HAL is a multi-disciplinary open access archive for the deposit and dissemination of scientific research documents, whether they are published or not. The documents may come from teaching and research institutions in France or abroad, or from public or private research centers.
L'archive ouverte pluridisciplinaire HAL, est destinée au dépôt et à la diffusion de documents scientifiques de niveau recherche, publiés ou non, émanant des établissements d'enseignement et de recherche français ou étrangers, des laboratoires publics ou privés. 


\section{CA I R N}

chercher : repérer : avancer

Cet article est disponible en ligne à l'adresse :

http://www.cairn.info/article.php?ID_REVUE=RFS\&ID_NUMPUBLIE=RFS 482\&ID_ARTICLE=RFS 482 0273

\section{La prostitution en clubs dans les régions frontalières de la République tchèque}

par Mathilde DARLEY

\section{| Ophrys | Revue française de sociologie}

\section{7/2 - Volume 48}

ISSN 0035-2969 | ISBN 978-2-7080-1174-8 | pages 273 à 306

\section{Pour citer cet article :}

- Darley M., La prostitution en clubs dans les régions frontalières de la République tchèque, Revue française de sociologie 2007/2, Volume 48, p. 273-306.

Distribution électronique Cairn pour Ophrys.

(c) Ophrys. Tous droits réservés pour tous pays.

La reproduction ou représentation de cet article, notamment par photocopie, n'est autorisée que dans les limites des conditions générales d'utilisation du site ou, le cas échéant, des conditions générales de la licence souscrite par votre établissement. Toute autre reproduction ou représentation, en tout ou partie, sous quelque forme et de quelque manière que ce soit, est interdite sauf accord préalable et écrit de l'éditeur, en dehors des cas prévus par la législation en vigueur en France. Il est précisé que son stockage dans une base de données est également interdit. 


\title{
La prostitution en clubs dans les régions frontalières de la République tchèque*
}

\begin{abstract}
RÉSUMÉ
Les espaces prostitutionnels dans les régions frontalières tchèques, apparus après l'ouverture des frontières du pays et essentiellement destinés à une clientèle germanophone, fournissent un terrain d'étude privilégié des configurations sociales et spatiales associées à la vente de services sexuels. Ils sont par ailleurs l'occasion de revenir sur les questions que posent à la sociologie l'accès à un terrain considéré comme «difficile » et la conduite d'entretiens avec les acteurs du milieu prostitutionnel. L'analyse des trajectoires prostitutionnelles des clients et des femmes prostituées dans les clubs des régions frontalières révèle le caractère complexe et multiforme des rapports de pouvoir qui s'y développent et, plus encore, le potentiel fantasmatique du fait social «prostitution ». Il semble alors que la prostitution frontalière en République tchèque puisse être lue comme un espace confiné de cristallisation des fantasmes et d'affirmation des identités.
\end{abstract}

La prostitution, définie ici comme «le fait d'établir avec d'autres personnes des relations dont l'aboutissement logique est un acte sexuel, avec pour but d'obtenir à court terme une rémunération »(Brochier, 2005), a fait son apparition dans les régions frontalières tchèques au début des années 1990, peu après la chute du régime communiste et l'ouverture des frontières du pays. Produits du différentiel économique entre la République tchèque et ses voisins ouest-européens, les relations sexuelles commercialisées se sont essentiellement développées à la frontière avec l'Allemagne et l'Autriche et s'adressent à une clientèle majoritairement germanophone. Les axes routiers reliant la République tchèque et l'Allemagne ou l'Autriche ont ainsi souvent été décrits comme des « routes du sexe », voire comme des «marchés aux esclaves » (1). Pourtant, le phénomène semble avoir longtemps fait l'objet, du côté tchèque, d'une forme de tabouisation sociale et scientifique. Le rapport publié peu avant l'entrée de la République tchèque dans l'Union européenne par l'organisation non gouvernementale allemande $K A R O$, qui dénonçait la présence d'une importante prostitution infantile à destination des clients

* L'auteur tient à remercier Gilles FavarelGarrigues, Christian Lequesne et Lilian Mathieu ainsi que les rapporteurs anonymes de la revue pour leur relecture attentive et leurs précieux commentaires sur les versions antérieures de cet article.

(1) Voir par exemple Florian Klenk, «Prostitution: der moderne Sklavenmarkt. Erkundungen im deutsch-tschechischen Grenzgebiet », Die Zeit, 40, 28/09/2006. 
allemands dans la ville de Cheb (Schauer, 2003), à la frontière avec l'Allemagne, a cependant déclenché une vive polémique autour du développement du tourisme sexuel dans les régions frontalières tchèques. Face à la résonance médiatique du phénomène, et après les déclarations des autorités tchèques et allemandes relativisant l'importance de l'exploitation sexuelle des enfants en République tchèque, l'intensification de la coopération policière entre les deux pays a été officiellement présentée comme la principale réponse au « problème ». Le vide juridique qui entoure les activités prostitutionnelles en République tchèque (2) et les obstacles qui subsistent en matière de coopération policière transnationale limitent néanmoins l'influence de l'institution policière sur le développement du commerce du sexe dans les régions frontalières. Ainsi, malgré la publication ponctuelle de quelques articles alarmistes par les journaux tchèques, allemands ou autrichiens sur le destin tragique des prostituées, migrantes notamment, et l'omniprésence du fait «prostitution » pour les populations frontalières, il semble plutôt que l'on feigne, de part et d'autre de la frontière, d'ignorer le phénomène, et les travaux portant sur la commercialisation des services sexuels aux frontières tchéco-allemande et tchéco-autrichienne restent rares. Le milieu prostitutionnel frontalier en République tchèque s'inscrit pourtant dans un espace géographique et social particulier, dont nous nous emploierons ici à rendre compte. La chute du régime communiste, l'histoire contrariée des frontières du pays jusqu'en 1993, son adhésion à l'Union européenne en 2004 et la simplification des contrôles frontaliers qui l'a accompagnée, mais aussi surtout les inégalités économiques persistantes entre la République tchèque et ses voisins allemand et autrichien sont autant d'éléments qui constituent la toile de fond de l'apparition et du développement de la sexualité prostitutionnelle dans le paysage frontalier tchèque. Ils attestent du rapport particulier entre frontière et prostitution: la frontière est ici l'institution qui rend possible certaines formes de commerce du sexe aux confins de la République tchèque, en raison de la juxtaposition de bas salaires et d'opportunités limitées pour les peu qualifiés (et en particulier pour les populations migrantes) d'un côté de la frontière, et de clients potentiels facilement accessibles et comparativement aisés de l'autre (Donnan et Wilson, 1999, p. 92).

(2) Sans être formellement autorisée, la prostitution n'est pas non plus prohibée en République tchèque. Si le droit tchèque interdit en principe l'ouverture d'établissements de prostitution, cette interdiction est aisément contournée par l'enregistrement officiel des établissements comme «pensions» ou «bars de nuit ». Un projet de loi visant à réglementer la prostitution a été déposé par l'ex-ministre de l'Intérieur tchèque František Bublan en août 2005. Il interdit la prostitution des mineurs de moins de 18 ans et prévoit l'obligation, pour les prostituées enregistrées, de se soumettre à des visites médicales régulières et de payer des impôts. Il augmente en outre les compétences des communes, qui pourraient décider des zones dans lesquelles l'activité prostitutionnelle est autorisée et interdire systématiquement la prostitution à proximité des écoles et des établissements de jeunesse. Les communes des régions frontalières de la République tchèque ont largement contribué à l'élaboration de ce projet de loi mais les chrétiens-démocrates et les conservateurs y restent majoritairement très hostiles. 
L'analyse présentée ici, qui résulte de recherches conduites en 2005 et 2006 sur la prostitution en clubs dans les régions frontalières de la Šumava (frontière tchéco-bavaroise) et de Moravie (frontière tchéco-autrichienne), a donc pour objectif d'étudier les relations entre différents acteurs de la prostitution, et notamment entre prostituées et clients, sous l'angle particulier de leur inscription spatiale à la frontière entre pays de niveaux économiques différents et à l'histoire politique contrastée. Il s'agira tout d'abord de chercher à éclairer le contexte géographique et socio-économique de la prostitution frontalière en République tchèque afin de mettre en relation l'organisation de la prostitution, les conditions qui la rendent possible et ses manifestations territoriales. Après avoir défini notre objet et contextualisé notre terrain de recherche, nous reviendrons sur la démarche méthodologique avec laquelle nous avons appréhendé un terrain traditionnellement étiqueté comme «difficile». Considérant le peu de recherches s'intéressant aux rapports de pouvoir à l'œuvre entre les acteurs du milieu prostitutionnel, nous tenterons ensuite de mettre en lumière les représentations et les trajectoires des acteurs de la prostitution frontalière, et notamment des clients et des prostituées, et les jeux de pouvoir traversant leurs relations. À partir de cette tentative de sociologie des acteurs, il semble alors possible de comprendre la prostitution comme un «construit social» (Howe, 2006), et les territoires prostitutionnels frontaliers comme des espaces symboliques d'affirmation et de réaffirmation des représentations identitaires.

\section{Caractéristiques géographiques et sociales des espaces prostitutionnels frontaliers}

Les régions frontalières étudiées se situent à l'ouest et au sud de la République tchèque (3) et se caractérisent avant tout par une concentration très importante, dans un espace relativement restreint, de «night clubs », appellation euphémisée des lieux de prostitution dans les régions frontalières tchèques. De vingt-cinq en 1997 , le nombre de clubs de prostitution est passé à plus de soixante-dix actuellement à la seule frontière tchéco-bavaroise (150 kilomètres) (4), pourtant bien moins exposée que la frontière du nord-ouest de la République tchèque. Les lieux de prostitution font donc partie intégrante du paysage, qu'ils dominent même parfois, comme dans certains villages dont la rue principale est bordée de clubs érotiques. Il importe cependant, pour comprendre les représentations et les stratégies d'acteurs, de replacer les formes de sexualité prostitutionnelle dans les zones frontalières tchèques dans le contexte historique, géographique et social particulier de leur développement.

(3) Les recherches ayant nourri ce texte ont plus particulièrement porté sur les localités de Folmava et Železná Ruda à la frontière tchécoallemande, et de Znojmo à la frontière tchécoautrichienne.
(4) Entretien avec les représentants d'une organisation de prévention sanitaire auprès des prostituées dans la région frontalière de Plzeň (frontière tchéco-bavaroise). 


\section{Ouverture des frontières et prostitution}

Apparue peu après l'ouverture des frontières de la Tchécoslovaquie puis de la République tchèque, la prostitution frontalière est de toute évidence née du différentiel économique important subsistant entre les régions frontalières allemandes ou autrichiennes et leurs pendants tchèques. Bien que certaines organisations non gouvernementales actives dans ces régions notent aujourd'hui une légère augmentation de la fréquentation des clubs par les hommes tchèques, ceux-ci ne représentent encore qu'une infime proportion des consommateurs de services prostitutionnels dans les zones frontalières, très majoritairement Allemands ou Autrichiens. Les législations nationales ne sauraient pourtant expliquer la quête, de la part des clients allemands et autrichiens, de services sexuels en République tchèque : si la prostitution est généralement considérée comme l'une des zones grises de la législation tchèque, elle est au contraire autorisée et réglementée en Allemagne et en Autriche. L'offre de services sexuels est certes inexistante dans les régions frontalières allemandes et autrichiennes, en raison notamment des restrictions qui s'appliquent à l'ouverture de lieux de prostitution en zone rurale (5), mais ces mesures paraissent avant tout avoir été pensées comme un obstacle à l'expansion des clubs de part et d'autre de la frontière, et constituent donc davantage un effet qu'une cause de la prostitution frontalière en République tchèque. Les prix pratiqués dans les lieux de prostitution frontaliers en République tchèque, généralement deux à trois fois inférieurs aux tarifs ayant cours dans les pays voisins, et la présence de biens et services annexes (essence, cigarettes, restauration, etc.), également meilleur marché, apparaissent donc comme les principaux facteurs explicatifs de la composition majoritairement germanophone de la clientèle (6).

L'attirail marketing très important développé par les propriétaires d'établissements de prostitution dans cet environnement hautement concurrentiel est donc quasi exclusivement en langue allemande et recourt aux arguments marchands «classiques » pour vanter les mérites de tel ou tel établissement : à la frontière tchéco-autrichienne, d'immenses affiches informent ainsi le visiteur de la possibilité qui lui est offerte d'avoir « du sexe aujourd'hui pour 39 euros » (Sex heute um 39 Euro), tandis qu'en devanture des clubs, des pancartes annoncent l'arrivée de « filles fraîches aujourd'hui » ou un approvisionnement «100\% tchèque ». Outre le recours à ces outils marketing habituellement réservés à d'autres «circuits de distribution », le registre sollicité par les propriétaires de clubs pour décrire la prostitution frontalière traduit souvent une représentation du milieu prostitutionnel en termes de « marché »,

(5) Voir la loi sur la prostitution (Gesetz zur Regelung der Rechtsverhältnisse der Prostituierten - ProstG) adoptée par le parlement allemand en décembre 2001 et entrée en vigueur le $1^{\text {er }}$ janvier 2002.

(6) L'organisation non gouvernementale Jana de prévention des maladies sexuellement transmissibles auprès des prostituées à la frontière tchéco-bavaroise estime à 2000 le nombre d'hommes allemands franchissant quotidiennement la frontière ouest de la République tchèque pour consommer des services sexuels dans les clubs de prostitution (prostitution de rue non incluse). Voir Roland Kirbach, «Die Rückkehr zum Leichtsinn », Die Zeit, 49, 25/11/2004. 
lieu de la rencontre de l'offre et de la demande de services sexuels. En témoigne par exemple la métaphore invoquée par un gérant pour justifier son refus de nous laisser interroger les clients sur le parking de son établissement : «C'est comme au supermarché, on ne peut pas se poster à l'entrée et dire aux clients que ce qui les attend à l'intérieur n'est pas bon. » (7). La concurrence porte ici non seulement sur le prix des services mais aussi sur les prestations offertes. On remarque ainsi, dans les zones frontalières, une forme de commercialisation accrue des services érotiques précédant ou accompagnant l'acte sexuel lui-même, tels que shows ou danses suggestives, dont la qualité semble constituer un argument marketing important pour les clients interrogés (8). S'ajoute à ces motivations commerciales la garantie de l'anonymat que paraît impliquer, pour les clients allemands ou autrichiens, le franchissement d'une frontière étatique.

Le recours à la prostitution transfrontalière comporte en effet également une dimension plus subjective, souvent inhérente au tourisme sexuel et sur laquelle nous reviendrons, qui consiste à construire les autres cultures, en l'occurrence les cultures dites «est-européennes», comme «différentes au regard des pratiques et des mœurs sexuelles » (Bishop et Robinson, 1998, p. 114). Le milieu de la prostitution frontalière en clubs se distingue en effet par la surreprésentation des prostituées issues d'anciennes Républiques socialistes (9), et notamment d'Ukraine et de la Slovaquie voisine, mais aussi de Russie, de Biélorussie, de Moldavie, ou encore de Roumanie et de Bulgarie. Cette prostitution étrangère est de toute évidence elle aussi le produit d'un différentiel économique, cette fois entre la République tchèque et les pays d'origine des prostituées. Ces dernières sont généralement dépeintes sous les traits de victimes d'organisations criminelles tirant profit de la prostitution de femmes migrantes, voire éventuellement d' "étrangères » aux mœurs dissolues et prêtes à tout pour de l'argent, mais leurs motivations et trajectoires de vie dans la prostitution restent finalement relativement inexplorées. Dans un contexte général de focalisation sur les questions de traite des femmes (10) et

(7) Citation d'un propriétaire de club à la frontière tchéco-autrichienne.

(8) Voir par exemple le site web d'un des principaux clubs de la région de Znojmo, à la frontière tchéco-autrichienne, où l'on peut lire : «Malheureusement, dans la plupart des lieux qui se présentent comme des night clubs et dont la superficie ne dépasse souvent pas $20 \mathrm{~m}^{2}$, on s'ennuie à mourir. Se divertir dans l'un de ces clubs peut ainsi se résumer uniquement à être assis devant la télévision. Fort heureusement, il existe également chez nous des night clubs qui méritent effectivement ce qualificatif et qui rendent la ville dans laquelle ils sont situés plus attractive. Dans notre région, le club X [nom modifié] est, sans exagération aucune, le meilleur lieu de divertissement de ce genre. »

(9) Selon les estimations de 1'ONG Jana, près de deux tiers des prostituées travaillant à la frontière tchéco-bavaroise sont issues d'anciens États socialistes. Voir les Actes du colloque de l'organisation Jana, AIDS und Prostitution in der erweiterten Union, altes Thema - neue Wege, Plzeň (République tchèque), octobre 2004.

(10) Voir les instruments internationaux récemment adoptés pour lutter contre la traite des femmes, et notamment le Protocole additionnel pour la prévention, la suppression et la répression de la traite des êtres humains, en particulier des femmes et des enfants, adopté le 15 novembre 2000 par l'Assemblée générale des Nations unies et ouvert à signature en décembre 2000 à Palerme, ou encore la Décision cadre relative à la lutte contre la traite des êtres humains adoptée par le Conseil de 1'Union européenne en juillet 2002. 
d'assimilation historique entre prostitution et traite des femmes (voir Corbin, 1978, pp. 405-406), les prostituées migrantes font l'objet de perceptions très différenciées selon les échelles d'analyse : si l'échelle internationale, où les énergies se concentrent sur la lutte contre la traite, est celle de l'indignation légitime et prône généralement une approche des prostituées migrantes en termes de « victimes », s'appuyant sur la médiatisation de cas de traite particulièrement dramatiques ou d'estimations alarmistes (11), le regard porté par les acteurs locaux sur les femmes migrantes exerçant la prostitution se fait plus facilement accusateur (Redoutey, 2005), comme en témoignent certaines déclarations recueillies auprès de la police, des populations locales ou encore des touristes dans les villages présentant une activité prostitutionnelle importante.

\section{Les territoires de la prostitution frontalière}

Les lieux de prostitution étudiés ici ne sont pas dédiés qu'à la prostitution, mais présentent au contraire des formes cycliques de territorialisation, leurs usages diurnes et nocturnes, mais aussi en semaine/week-end, variant très fortement. Les manifestations territoriales du caractère cyclique de l'activité prostitutionnelle diffèrent cependant de celles observées dans d'autres contextes, et notamment dans le paysage urbain de Rio de Janeiro (Botelho de Mattos et Angelo Campos Ribeiro, 1997). Le village de Železná Ruda, dans le massif de la Śumava au sud-ouest de la République tchèque, est essentiellement fréquenté, en journée, par des touristes randonneurs en été et amateurs de ski en hiver, tandis que la région de la Moravie, au sud, est réputée pour ses activités viticoles et tente de promouvoir un tourisme vert. En outre, les régions frontalières tchèques accueillent également d'immenses marchés vietnamiens, sur lesquels nous reviendrons, spécialisés dans le commerce de produits de contrefaçon et très prisés des populations frontalières allemandes et autrichiennes. Par ailleurs, certaines structures développées au cours des dix dernières années du côté tchèque de la frontière, tel l'énorme centre commercial d'Excalibur City (proposant wellness, magasins, centre de soins pour animaux, crèche, station service et un service de bus quotidien depuis la capitale autrichienne), sont venues enrichir encore l'offre des régions frontalières tchèques, en matière de loisirs et de tourisme, pour les pays voisins germanophones. Plus classiquement, l'installation de casinos est également associée, de manière quasiment systématique, aux établissements de prostitution, de nombreux clients ayant pour habitude de débuter la soirée au casino et de la terminer dans un club. La position géographique des territoires prostitutionnels frontaliers sur d'importants axes routiers reliant par exemple Dresde et Prague pour les régions du nord, Plzeň et Munich ou Salzbourg au sud-ouest ou encore Prague et Vienne au sud a, elle aussi, favorisé cette

(11) Voir à titre d'exemple les déclarations de Detlef Ubben, chef du département Traite des êtres humains à l'Office criminel de Hambourg, qui estime à $95 \%$ la part des prosti- tuées contraintes. Cité dans Florian Klenk, «Prostitution: der moderne Sklavenmarkt. Erkundungen im deutsch-tschechischen Grenzgebiet », Die Zeit, 40, 28/09/2006, p. 4. 
superposition de différentes activités touristiques, commerciales et prostitutionnelles dans les zones frontalières tchèques.

En dépit de l'importance, sur les portions frontalières de ces grands axes routiers, de la prostitution de rue, nous avons choisi de nous concentrer ici sur la prostitution en clubs. Ce choix procède de plusieurs facteurs : d'une part, l'étude de la prostitution de rue n'aurait pas permis d'approcher de manière aussi systématique les clients, qui ne descendent généralement pas de leurs véhicules lorsqu'ils recourent aux services des prostituées postées sur le bord des axes routiers transfrontaliers ; d'autre part, le milieu de la prostitution de rue est réputé plus difficile à pénétrer en raison notamment de la mainmise de quelques groupes, souvent familiaux et d'origine rom, sur les activités des prostituées, qui sont de ce fait très contrôlées (12). Par ailleurs, la prostitution de rue est moins importante dans les régions étudiées ici que dans les zones frontalières du nord-ouest de la Tchéquie, traversées par la route E55 désormais connue pour ses allures de marché du sexe de plein air. Les hypothèses présentées dans cet article ne sauraient donc s'appliquer à l'ensemble de la prostitution frontalière en République tchèque, le cas de la prostitution de rue et des régions frontalières du nord-ouest du pays devant sans doute faire l'objet d'une étude spécifique.

Il apparaît possible de dégager, sur la base des entretiens conduits et des visites effectuées dans certains lieux de prostitution, une forme de « géographie prostitutionnelle (Redoutey, 2005) tenant compte de la localisation des clubs, mais aussi de leur taille et de leur volume d'activité. Ainsi, tandis que le bourg de Železná Ruda, situé à la frontière avec la Bavière, présente une forte concentration des clubs en centre-ville, en bordure de la rue principale, les clubs installés dans la région de Znojmo (localité à la frontière tchéco-autrichienne) sont au contraire dispersés dans un rayon de plusieurs kilomètres autour de la ville. Cette localisation différenciée semble influer, dans une certaine mesure, sur les modes de vie et de travail des prostituées ainsi que sur leur rapport à la population locale : les prostituées travaillant et vivant dans les clubs de Železná Ruda sont connues des habitants, des commerçants et des chauffeurs de taxi, qui indiquent spontanément le bar où elles ont l'habitude de se réunir l'après-midi, aux moments où l'activité est plus réduite. Le caractère non anonyme de leur présence peut donc éventuellement constituer une forme de sécurité pour les femmes prostituées. Au contraire, en raison de la situation géographique relativement isolée des clubs dans la région de Znojmo (Moravie), les femmes peuvent y être plus dépendantes des gérants d'établissements, qui accompagnent généralement en voiture chacune de leurs sorties.

Par ailleurs, les lieux de prostitution présentent des caractéristiques de taille, et donc d'activité, très différentes d'une ville à l'autre mais aussi d'un club à l'autre : les lieux les plus connus sont en général de taille importante, emploient trente à quarante filles environ, réputées très belles, et l'agencement

(12) Constat également dressé par les ONG d'aide aux prostituées, qui peinent parfois à approcher les prostituées de rue. 
des salles témoigne d'une marchandisation «professionnelle » des services sexuels, avec intérieur rouge, pistes de danse, jeux de lumière et aménagements luxueux (piscines, jacuzzi, etc.). Dans ces clubs, plusieurs filles en sous-vêtements se pressent autour du client à son entrée et il semble que l'on ait cherché à créer une ambiance propice à la consommation de services sexuels. Il existe cependant également des clubs à la taille beaucoup plus modeste, employant un petit nombre de prostituées (deux ou trois filles pour les clubs les moins importants) et dont l'intérieur rappelle, selon les clubs, un salon de maison privative ou un bar de dernière catégorie.

\section{Modalités et acteurs de la rencontre prostitutionnelle}

Les quatre prostituées employées d'un club de petite taille à la frontière tchéco-allemande ont indiqué une fréquentation moyenne de cent vingt clients entre samedi et dimanche contre soixante environ sur le reste de la semaine, confirmant l'écart important constaté sur le terrain entre le calme relatif des jours de semaine et la suractivité enregistrée pendant le week-end. Les observations effectuées dans les clubs et à leurs abords, mais aussi les entretiens réalisés avec des prostituées et des clients de la prostitution ont mis en évidence la possibilité d'un clientélisme «passif» parmi les hommes fréquentant les établissements de prostitution: nous désignons ainsi le comportement des hommes se rendant de manière plus ou moins régulière dans les clubs de prostitution sans pour autant nécessairement consommer des services sexuels. Cette forme de fréquentation des lieux de prostitution peut constituer une première étape avant le développement d'un clientélisme " actif », avec consommation de services sexuels, ou bien correspondre à un mode durable d'utilisation des établissements de prostitution, alors davantage envisagés comme lieux de spectacle, où l'on vient «voir », ou de rencontre, où l'on vient avant tout établir un contact, éventuellement un flirt.

Les passes, quand elles ont lieu, sont réalisées dans les chambres, qui constituent à la fois l'espace privé des prostituées (elles sont hébergées dans les clubs, où elles partagent fréquemment une chambre avec une collègue) et leur lieu de travail. Contrairement aux clubs allemands et autrichiens, qui fixent des règles claires de tarification associant à un prix à la fois un minutage de la passe et une délimitation des services offerts (strip-tease et rapport vaginal par exemple), le tarif de la passe dans les clubs des régions frontalières tchèques est généralement fixé en fonction de sa durée et non des prestations offertes. Les organisations non gouvernementales insistent sur le caractère attractif de ce mode de facturation pour les clients allemands ou autrichiens : les femmes travaillant en clubs dans les régions frontalières, à plus forte raison quand elles sont étrangères, semblent peiner à imposer des limites à leur partenaire concernant les services sexuels auxquels il peut prétendre, et celui-ci peut donc «tout se permettre»(13).

(13) Entretien avec les représentants d'une organisation de prévention sanitaire auprès des prostituées à la frontière tchéco-bavaroise. 
La chambre se négocie généralement entre trente et quarante euros pour une demi-heure, et les prestations les plus demandées semblent être la fellation, que les clients disent ne pouvoir obtenir de leurs partenaires habituelles, et le rapport vaginal. Certains clubs ont mis en place, pour répondre à la demande des clients plus jeunes, des tarifs spéciaux pour les services sexuels « rapides », facturés de vingt à trente euros pour un quart d'heure (14). Les modalités de paiement de la passe varient d'un club à l'autre : le client paye la passe soit au bar, c'est-à-dire au gérant, soit directement à la prostituée, qui reverse ensuite une partie de ses gains au gérant. La répartition des bénéfices entre propriétaires des clubs et prostituées peut prendre deux formes: les clubs les plus importants choisissent généralement un système de paiement forfaitaire, en vertu duquel les prostituées payent une somme fixe, à la journée ou au mois, destinée à couvrir les frais liés à leur hébergement. Ce «loyer» payé, elles peuvent ensuite conserver l'intégralité de l'argent qui leur est remis par les clients, mais doivent cependant financer seules leurs dépenses alimentaires et les consommations au bar, généralement surfacturées, inhérentes à leurs activités dans le club (15). C'est également ce système de versement forfaitaire que semblent privilégier les clubs les plus modestes, en raison de l'entrée d'argent régulière qu'il garantit au propriétaire. Au contraire, les clubs de taille moyenne optent en général pour un système d'hébergement «gratuit», en échange duquel la prostituée remet $50 \%$ du montant de chaque passe au propriétaire. À ces modalités de répartition des bénéfices, qui conduisent généralement les prostituées à reverser, sous une forme ou une autre, environ la moitié de leurs gains au propriétaire du club, s'ajoute éventuellement, pour les Ukrainiennes notamment, le versement d'une partie de leurs bénéfices au proxénète qui a arrangé leur voyage vers la République tchèque et leur placement en club. Celui-ci entretient en effet des relations privilégiées avec certains clubs, qu'il approvisionne en filles et autour desquels il gravite, et exige en règle générale que lui soient remis, par la prostituée, $25 \%$ de ses gains, pour le remboursement des frais qu'il dit avoir engagés pour l'obtention du visa et l'organisation du voyage. Les prostituées ukrainiennes ne conservent donc généralement qu'un quart du total de leurs gains.

Les propriétaires de clubs constituent un acteur du milieu prostitutionnel difficile à étudier. Cependant, un long entretien conduit avec un propriétaire venu en client dans l'un des clubs considérés ici, certaines déclarations recueillies auprès des prostituées et des clients, les observations collectées au sein des clubs et les renseignements délivrés par les acteurs non gouvernementaux fournissent quelques éléments d'information. Si l'on en croit la police et les organisations non gouvernementales, il semble que la plupart des propriétaires officiels de clubs soient de nationalité tchèque, en raison notamment des restrictions imposées par le droit tchèque à l'acquisition de biens de propriété par les étrangers, mais travaillent en coopération avec un partenaire

(14) Informations recueillies auprès de prostituées travaillant à la frontière tchécoallemande.
(15) Entretiens avec des prostituées en clubs à la frontière tchéco-allemande. 
allemand ou autrichien. Bien que peu de propriétaires aient toléré la présence d'enquêteurs sur le parking de leur club, certains ayant même réagi avec violence et par des menaces à notre simple demande d'autorisation, la plupart d'entre eux acceptent les visites régulières des organisations non gouvernementales habilitées à vérifier dans les clubs l'état de santé des femmes y travaillant, et notamment à détecter la présence d'éventuelles maladies sexuellement transmissibles. La présence de filles «saines » est en effet un élément important de l'image des clubs, et les gérants déclarent d'ailleurs souvent préférer les filles tchèques ou slovaques aux «étrangères », reprochant à ces dernières, et notamment aux prostituées ukrainiennes, une consommation d'alcool excessive (16). Nombre d'entre eux fondent leurs outils de marketing sur certains préjugés racistes en vertu desquels les femmes dites «est-européennes », en tête desquelles ils placent les femmes tchèques, seraient plus belles, plus «fraîches », moins arrogantes mais aussi plus portées sur le sexe que les femmes d'Europe de l'Ouest. Ces stéréotypes culturalistes se doublent souvent d'une représentation sexuée des rôles sociaux, en vertu de laquelle la prostitution serait « le meilleur travail qu'une femme puisse faire si elle veut gagner de l'argent », ce qui expliquerait d'ailleurs qu'il s'agisse là du «plus vieux métier du monde » (17). Un jeune propriétaire d'établissements de prostitution interrogé à la frontière tchécoallemande affirme ainsi : «Si une femme n'a pas de problème avec ça, elle serait bête de ne pas faire ce travail. De toutes façons, les femmes n'ont jamais de relations libres avec les hommes. Quand elles ont un petit ami, il faut qu'il paye pour l'appartement ou la voiture, sinon il n'est plus intéressant. Donc ça n'a rien de surprenant que des femmes décident de faire payer pour leur corps. » Ce propriétaire décrit par ailleurs les principaux clubs des régions frontalières tchèques comme très professionnels, respectant les normes d'hygiène et sélectionnant soigneusement les filles autorisées à y travailler. L'ambiance y est selon lui «calme et décontractée » et les femmes sont «toutes volontaires ». Les contrôles policiers sont relativement rares, et les propriétaires semblent entretenir de bons rapports avec les fonctionnaires de police (18), qui déclarent qu'«il n'y a pas de problème » (19).

Enfin, bien que les modalités de la rencontre prostitutionnelle en extérieur (de rue) ne soient pas abordées ici et n'aient pu faire l'objet de recherches approfondies, certains aspects peuvent en être mentionnés afin de donner à voir les particularismes liés aux différentes formes de prostitution dans les régions frontalières. Quoique beaucoup plus développée aux frontières nordouest de la République tchèque (Bohême du Nord), la prostitution de rue est également présente, mais de manière plus marginale, dans les régions de la

(16) Entretien avec les membres d'une ONG d'aide aux prostituées à la frontière tchéco-allemande.

(17) Citations extraites d'un entretien avec un propriétaire de club allemand à la frontière tchéco-allemande.

(18) Les fonctionnaires de police ont parfois été accusés d'entretenir des liens étroits avec les propriétaires d'établissements de prostitution. Voir par exemple les déclarations recueillies auprès des fonctionnaires de la cellule de lutte contre le crime organisé à Plzeň et Brno.

(19) Entretien avec des fonctionnaires de la police municipale à la frontière tchécoallemande. 
Šumava et de Moravie étudiées ici. Elle concerne quasi exclusivement des femmes tchèques (Šumava) et roms (Moravie) et est généralement décrite par les acteurs non gouvernementaux locaux comme plus dangereuse pour les prostituées. Celles-ci y sont en effet davantage soumises au contrôle policier, plus aisé en raison de la visibilité et de l'accessibilité des femmes exerçant la prostitution en extérieur, et aux agressions, tant de la part des clients que de celle de «gangs » se livrant au racket des prostituées (20). Les prostituées interrogées semblent elles-mêmes opérer une hiérarchisation des formes de l'activité prostitutionnelle plaçant la prostitution de rue en bas de l'échelle, en raison non seulement des tarifs généralement inférieurs qui y sont pratiqués, mais aussi des conditions plus difficiles d'exercice de l'activité et de la présence de femmes contraintes à la prostitution par différentes formes de dépendance, aux drogues notamment.

\section{Interroger les acteurs de la prostitution : clients et prostituées}

Les axes de réflexion présentés dans cet article sont nés des entretiens conduits avec différents acteurs du milieu prostitutionnel, et notamment des clients germanophones de la prostitution frontalière en République tchèque et des prostituées exerçant en clubs, des représentants d'organisations non gouvernementales en charge du travail social avec les prostituées dans les régions frontalières, des fonctionnaires de police, et des habitants des villages considérés comme des centres de prostitution. L'accent a cependant été mis sur les clients de la prostitution et leur rapport à la femme prostituée ainsi que, de manière moins systématique, sur les prostituées elles-mêmes. Comme il a été indiqué plus haut, les «exploitants », propriétaires ou gérants des clubs, n'ont été abordés que marginalement, soit lors de la prise de contact en vue d'une demande d'autorisation d'enquêter dans les clubs, soit lorsque certains d'entre eux ont été approchés sur les parkings des clubs comme de simples clients.

\section{«Faire parler » les clients}

La diversité des acteurs interrogés nous a naturellement conduit à adapter la méthodologie employée aux différentes familles d'enquêtés. Si le recueil d'informations de la part de la population locale s'est révélé assez aisé et a généralement pris la forme de conversations informelles avec les employés des hôtels, bars et restaurants de la région ainsi qu'avec les personnes domiciliées à proximité immédiate des établissements de prostitution, la collecte d'informations auprès des clients de la prostitution est habituellement réputée difficile, ce qui explique que les études portant spécifiquement sur les

(20) Problème évoqué notamment par les organisations d'aide aux prostituées dans les régions frontalières. 
consommateurs de services sexuels soient encore très marginales. Nous avons pris le parti d'aborder ce terrain de recherche sans avoir défini de méthodologie particulière, en raison d'une part des avis très partagés recensés dans la littérature concernant la «meilleure manière d'aborder les clients », et d'autre part du sentiment qu'arriver sur ce terrain dit «difficile» munie d'une batterie de préceptes méthodologiques présentait le risque d'enfermer l'objet, de le catégoriser comme « difficile » avant même d'en avoir personnellement fait l'expérience.

Nous avons tout d'abord choisi d'aborder les clients à proximité immédiate des lieux de prostitution et aux moments de fréquentation maximale, c'est-àdire essentiellement la nuit et de préférence le week-end (21). En effet, les premières tentatives d'approche, réalisées en plein jour dans les stations services des régions frontalières tchèques (où les Allemands et les Autrichiens domiciliés de l'autre côté de la frontière viennent régulièrement faire le plein d'essence pour un prix inférieur à celui pratiqué dans les stations allemandes ou autrichiennes), ont révélé la réticence souvent indignée des hommes coutumiers du «shopping transfrontalier» à parler de sexe et de prostitution en «terrain neutre». Ces premiers entretiens nous ont donc permis de constater l'importance de l'établissement d'une distinction entre deux mondes, diurne (le monde des excursions, éventuellement en famille, dans les bazars vietnamiens de l'autre côté de la frontière, agrémentées d'un passage par la pompe à essence et le distributeur de cigarettes) et nocturne, possiblement réservé au tourisme sexuel.

Afin de ne pas heurter les sensibilités personnelles des hommes interrogés, nous avons ensuite entrepris de présenter notre enquête de manière relativement neutre, voire naïve, comme une recherche académique portant sur les régions frontalières en général et au cours de laquelle il nous serait apparu que les night clubs occupent une place importante dans le paysage frontalier. Sans présupposer du recours des personnes interrogées au sexe prostitutionnel, alors même que nous les avions vues entrer ou sortir d'un club, nous avons dans un premier temps préféré leur demander si «par hasard» ils connaissaient un peu la situation, éventuellement par ouï-dire. La conduite de premiers entretiens exploratoires débutant par des questions portant sur la connaissance des personnes interrogées du milieu de la prostitution avait en effet révélé la tendance de nos interlocuteurs à interpréter la simple prononciation du mot «prostitution», au cours de la prise de contact, comme une mise en accusation de pratiques sexuelles personnelles éventuellement

(21) Pour les besoins de cette enquête, environ vingt-cinq clients germanophones ont été interrogés, en Allemand, et la durée des entretiens varie de 10 à 90 minutes. Les entretiens ont été réalisés au cours de quatre séjours de terrain dans les régions frontalières considérées, d'une durée moyenne de trois à cinq jours chacun. En raison des conditions matérielles de réalisation des entretiens (de nuit et sur des parkings), ceux-ci n'ont pu donner lieu ni à un enregistrement ni à une prise de notes simultanée. Les informations recueillies ont donc été regroupées a posteriori sous la forme d'un cahier de notes ethnographiques, complété de manière succincte après chaque entretien et de manière plus détaillée après chaque série d'entretiens. 
stigmatisées. Les réactions collectées se cantonnaient alors quasiment exclusivement au registre de l'indignation, voire à un refus catégorique de se prononcer sur la question. C'est donc sur la base de cette première expérience que nous avons pris conscience de la nécessité de bannir les formulations pouvant impliquer la consommation de services sexuels par nos interlocuteurs, laissant à ces derniers le soin de révéler, s'ils le souhaitaient, leur rôle de client actif de la prostitution. Nous avons ensuite privilégié, dès le début de l'entretien, les questions portant non sur les clients eux-mêmes et leurs pratiques sexuelles, mais sur les femmes travaillant en clubs, afin de donner une dimension moins « confessionnelle » aux informations livrées par les hommes interrogés. Aborder la question de la prostitution par le biais des prostituées présente en effet l'avantage de laisser toujours à la personne interrogée, si elle le souhaite, la possibilité de présenter les informations et les impressions qu'elle nous livre comme étant « de seconde main » et de conserver ainsi le sentiment de protéger son intimité (même si la précision des informations livrées témoigne souvent elle-même d'une connaissance très exacte du milieu prostitutionnel à laquelle des informations de seconde main ne suffiraient sans doute pas). Nous avons ici considéré comme clients tous les hommes interrogés sur les parkings des clubs à leur entrée ou leur sortie du club. Bien que nombre d'entre eux aient indiqué ne pas consommer de services sexuels, la connaissance relativement étendue qu'ils semblaient avoir du fonctionnement des lieux et le caractère régulier de leurs visites nous ont conduits à recueillir leur témoignage comme celui d'un client.

Le choix, par tâtonnements successifs, de la question des prostituées comme une des techniques d'approche des clients permet par ailleurs de faire vibrer chez les clients la "corde héroïque » en évoquant le rôle de sauveteur (d'une femme forcée à se prostituer) qu'ils pourraient éventuellement être amenés à jouer dans le jeu prostitutionnel. Nous aurons l'occasion de revenir sur l'importance, dans les discours des clients de la prostitution, de cette dimension héroïque, mais signalons pour l'instant que son utilisation pour l'établissement d'un contact avec les clients semble avoir d'emblée permis de nous distinguer, aux yeux des clients, d'une rhétorique globalisante et moralisatrice. Il était d'autant plus important de communiquer rapidement au client cette prise de distance à l'égard de la perception généralement admise des clients comme misogynes que l'enquêteur était de sexe féminin et courait donc le risque d'être a priori assimilé par les personnes interrogées aux différents groupes de lobby féministes condamnant le recours des hommes à une forme de sexualité prostitutionnelle (22). Nous avons donc cherché à mettre en avant notre effort de neutralité et d'absence de parti pris, nous appuyant pour ce faire sur une formulation conditionnelle et, dans la mesure du possible, impersonnelle des questions. Les circonstances dans lesquelles ont été conduits les entretiens, à savoir de nuit et sur les parkings des lieux de prostitution, c'est-à-dire dans un «espace-frontière » entre l'intimité des

(22) Voir par exemple le rapport de la Coalition Against Trafficking in Women $(C A T W)$, disponible sur http://www.catwinter- national.org/factbook/czech.php (consulté le 04/08/2006). 
clubs et l'anonymat de l'espace public qui commence dès la sortie de l'enceinte du parking, ont également pu contribuer à éviter l'instauration d'un rapport de force entre l'enquêteur et l'enquêté, tous deux se trouvant alors dans une zone liminaire et incertaine dont la pénétration est habituellement associée à certains risques pour les deux parties. Il convient toutefois de garder à l'esprit que, dans la mesure où nous n'avons pu nous entretenir qu'avec les clients disposés à parler, nous n'avons sans doute pas rencontré les clients considérés comme dangereux par les prostituées elles-mêmes ou, si nous les avons rencontrés, ceux-ci ont pris soin de taire ceux de leurs comportements qu'ils savent porter atteinte à l'intégrité physique ou psychologique de la personne prostituée. Les hypothèses présentées ici ne prétendent donc aucunement à une forme de représentativité, illusoire dans toute recherche sur les clients de la prostitution, mais résultent d'informations recueillies au cours d'une observation ethnographique des territoires et des acteurs prostitutionnels étudiés.

Nous avons d'abord craint que les clients se refusent à aborder leur sexualité prostitutionnelle avec une interlocutrice de sexe féminin et éventuellement plus jeune qu'eux. Pourtant, il semble que l'âge et le sexe de l'enquêteur n'aient finalement pas constitué un obstacle au recueil des confidences des clients, et aient même dans certains cas représenté une ressource (23) : en effet, tandis que la proximité d'âge encourageait les plus jeunes à utiliser, dans leurs réponses, le registre de la complicité générationnelle, les plus âgés développaient souvent à notre encontre une forme de paternalisme sans doute attisée par le caractère volontairement naïf des questions.

\section{L'échange avec les prostituées}

Les entretiens avec les prostituées travaillant en clubs (24) ont majoritairement été conduits à proximité des clubs mais hors de leur enceinte (dans des espaces publics tels que les arrêts de bus, etc.) et aux « heures creuses », et notamment en semaine l'après-midi, où les clients se font les plus rares. Certaines conversations ont également pu être engagées dans les clubs dont l'entrée nous a été autorisée et où l'activité était alors suffisamment réduite pour permettre de s'entretenir avec les rares clients et les prostituées (25).

(23) D’autres enquêtes réalisées sur les clients de la prostitution font également état de la préférence affichée par les consommateurs de services sexuels pour les entretiens réalisés avec un enquêteur de sexe féminin, en raison de la plus grande facilité avec laquelle ils disent se confier à une femme. Voir par exemple Bouamama (2004, p. 154).

(24) Pour les besoins de cette enquête, seuls six entretiens ont été conduits avec des prostituées, l'accent portant davantage sur le rapport du client à la prostituée. Les entretiens ont duré en moyenne entre deux et trois heures et les informations recueillies auprès des prostituées ont été confirmées et éventuellement complétées par les entretiens conduits avec des représentants d'ONG d'aide aux prostituées ainsi qu'avec la police locale.

(25) Dans le cas des entretiens en extérieur, le contact ayant été établi de manière relativement informelle avec un groupe de prostituées qui prenaient un bain de soleil à un arrêt de bus, nous avons souhaité conserver la tonalité informelle de l'entretien en ne recourant pas à 
Le contact avec les femmes travaillant en clubs paraît avoir été facilité par le fait que l'enquêteur ait été de sexe féminin et sensiblement du même âge que les femmes interrogées. Certes, la faible différence d'âge rend quasiment inévitable, de la part des prostituées, la comparaison de leur situation avec celle de l'enquêteur. Cependant, bien qu'il soit vain de chercher à nier qu'une telle comparaison encourage souvent, chez la prostituée, la perception de son propre sort comme négatif, il nous semble qu'elle a également permis d'identifier certaines des stratégies de résistance des prostituées au stigmate qui accompagne l'exercice de leurs activités. Les entretiens conduits à l'extérieur des clubs se sont déroulés de manière tout à fait libre avec un groupe de prostituées dont notre présence dans le village avait éveillé la curiosité. Pour assouvir cette curiosité, nous avons veillé à permettre aux femmes interrogées de nous poser autant de questions qu'elles le souhaitaient. Ces questions se sont essentiellement concentrées sur l'âge et le parcours personnel et professionnel de l'enquêteur et les réponses apportées ont souvent d'abord suscité, par l'effet de comparaison mentionné ci-dessus, une première réaction de dévaluation par les prostituées de leur propre expérience. À ce regard résigné et négatif adopté sur leur propre condition s'est pourtant rapidement substituée une tentative de valorisation, verbale mais aussi comportementale, de certains des aspects de leur activité ou des compétences qu'elle requiert. Nous développerons ci-après ces stratégies, que nous comprenons généralement comme des vecteurs de "résistance au stigmate", et qui ont pu d'autant mieux émerger que les entretiens ont été menés en extérieur et sans contrainte spatio-temporelle pour les enquêtées (qui allaient et venaient entre le club et l'espace dans lequel nous avions pris place) et que l'accent a été mis, au cours de la conversation, sur les rapports des prostituées à leurs clients : en effet, cet aspect de leur activité, que les organisations non gouvernementales en contact avec les prostituées évitent souvent délibérément, nous semble permettre de remettre en question certaines représentations stéréotypées des rapports de force à l'œuvre dans le milieu prostitutionnel.

\section{Trajectoires prostitutionnelles}

Les informations recueillies auprès de ces différents acteurs révèlent un jeu d'influences et de pouvoir plus complexe que ne le laissent généralement entrevoir les travaux sur la prostitution. Afin de mettre en lumière cette complexité, nous chercherons ici à rendre compte des pratiques et discours qui, chez les clients et les prostituées, accompagnent l'expérience du milieu prostitutionnel.

(suite note 25)

l'enregistrement. Les échanges ont toutefois donné lieu à une prise de notes simultanée, complétée ultérieurement. Les entretiens réalisés en clubs n'ont bien sûr pas pu faire l'objet d'un enregistrement ou d'une prise de notes en raison de la présence de clients potentiels. Ils ont donc, comme les entretiens avec les clients, donné lieu à la rédaction d'un cahier de notes à la sortie du club. 


\section{Les clients, perdants ou pervers?}

La fascination généralement exercée par les prostituées sur les auteurs, écrivains (Siegmund, 2006) ou chercheurs, tend à laisser dans l'ombre les hommes clients de la prostitution (Hoigard et Finstad, 1992). Le manque de données empiriques sur les pratiques et les représentations prostitutionnelles des clients est souvent justifié par la difficulté d'interroger cette famille d'acteurs et encourage alors sa perception comme un groupe homogène au comportement sexuel déviant et marginal. Les entretiens conduits avec des représentantes d'organisations non gouvernementales d'aide aux prostituées révèlent d'ailleurs que les acteurs directement impliqués dans le champ prostitutionnel n'échappent pas non plus à certaines peurs diffuses et au sentiment général qu'il leur faut se protéger et garder leurs distances vis-à-vis des hommes consommateurs de services sexuels (Howe, 2004). Espérant ainsi rendre compte d'une présence nébuleuse et mal connue, certains sociologues ont tenté de dresser une typologie des clients et de leurs motivations en les regroupant en catégories (Månsson, 2006) : celles-ci distinguent généralement les clients sexuellement attirés par l'image de la «putain », ceux qui recherchent dans la prostitution des formes de sexualité auxquelles ils n'ont pas accès avec leurs partenaires sexuelles habituelles, ceux pour lesquels la sexualité prostitutionnelle constitue la seule forme de sexualité possible, les hommes qui voient dans l'acte sexuel une nécessité physique justifiant certaines pratiques de consommation, parmi lesquelles la prostitution présente l'avantage de ne pas requérir d'investissement émotionnel (Prieur et Taksdal, 1993), ou encore les clients nostalgiques d'un ancien ordre de domination masculine sur les femmes (Welzer-Lang, Barbosa et Mathieu, 1994, p. 195). Cette catégorisation, établie par divers auteurs dans des contextes nationaux différents (France et Suède par exemple), est également celle qui a inspiré la typologie proposée par Saïd Bouamama (2004), distinguant essentiellement quatre types de clients : les isolés affectifs et sexuels, les décalés de l'égalité (recherchant une image soumise de la femme), les acheteurs de marchandises et les allergiques à l'engagement et à la responsabilité. D'autres typologies reposent au contraire davantage sur les habitudes de consommation sexuelle que sur les motivations du client et distinguent les clients accidentels des clients réguliers et, parmi ces derniers, les clients ayant des relations avec d'autres femmes des clients vivant seuls (Mossuz-Lavau, 2005).

Il est effectivement possible d'identifier, dans les discours que nous avons recueillis auprès des clients, certains des éléments censés sous-tendre ces catégorisations. Pourtant, l'autorité qui semble se dégager des typologies établies, lesquelles structurent la plupart des travaux portant sur les clients de la prostitution, peut conduire à ne retenir des informations communiquées par le client que les éléments corroborant les catégories considérées comme légitimes et réduire ainsi le champ des possibles dans l'analyse des motivations et des pratiques de la sexualité prostitutionnelle. C'est pourquoi nous ne chercherons pas ici à dresser une nouvelle typologie des clients de la prostitution ou à confirmer une typologie déjà établie, mais simplement à rendre compte 
de certains modes discursifs de rationalisation d'une sexualité généralement considérée comme déviante, sans pourtant prétendre à l'exhaustivité : d'une part, l'analyse des discours, en focalisant l'attention sur celui qui énonce, fait courir le risque de négliger certaines techniques de pouvoir à l'œuvre dans les mécanismes de recours à la prostitution; d'autre part, les résultats des entretiens conduits avec les clients, quelle que soit la taille de l'échantillon, ne sauraient être considérés comme représentatifs en raison de l'impossibilité qui subsiste d'interroger certains clients (26).

Dans les régions frontalières de la République tchèque, le recours à une sexualité prostitutionnelle est largement interprété par les hommes revendiquant leur statut de non-clients, mais aussi par les populations locales, et même par les prostituées elles-mêmes (27), comme le signe de l'appartenance à la catégorie des " perdants », tant au jeu économique qu'à celui de l'amour : les consommateurs de services sexuels marchands sont assimilés aux chômeurs vivant des aides sociales allemandes ou autrichiennes et qui « doivent payer pour ce que d'autres peuvent avoir gratuitement» (28), c'està-dire pour une partenaire sexuelle. Face aux jugements moralisateurs généralement portés sur leurs habitudes sexuelles, les clients autrichiens et allemands de la prostitution frontalière en clubs développent différentes stratégies discursives allant du déni au dédouanement en passant par l'héroïsation de leurs pratiques sexuelles.

La plupart des clients ont intériorisé le stigmate inhérent aux formes de sexualité prostitutionnelle et se placent tout d'abord dans une position de déni par rapport à toute éventuelle implication dans le milieu prostitutionnel, alors même qu'ils sont interrogés à l'entrée ou à la sortie des clubs et que la régularité de leurs visites est attestée par le gardien du parking par exemple. Les arguments alors avancés pour persuader l'enquêteur de sa non-appartenance au « groupe » des clients témoignent de la perception par le client lui-même de cette forme de sexualité comme négative et déviante. L'un de ses premiers réflexes est en effet de chercher à replacer immédiatement son comportement sexuel et amoureux dans la «normalité », ou ce qui est généralement perçu comme tel, au moyen d'arguments apparemment pensés comme automatiquement « disculpants », parmi lesquels figure notamment l'affirmation suivante : « Je ne suis pas client, j'ai une petite amie » (29). Pour bien signifier à l'interlocuteur une coupure avec le monde déviant de la sexualité prostitutionnelle, certains hommes refusent également de poursuivre l'entretien sur ce sujet. Pourtant, dans la majorité des cas, les personnes que nous avons interrogées étaient prêtes à livrer leurs impressions sur le milieu de la prostitution après s'en être clairement affranchies auprès de l'enquêteur: ayant consacré les premières phrases de l'entretien à établir son statut de non-client et à justifier

(26) Les citations ponctuant le texte ont été, en l'absence de référence particulière, extraites des entretiens conduits avec les clients germanophones de la prostitution en clubs en République tchèque et traduites par l'auteur.

(27) Voir plus loin.
(28) Citation d'un homme se définissant comme non-client.

(29) Réponse formulée par plusieurs hommes interrogés à l'entrée ou à la sortie des clubs. 
sa présence dans les lieux de prostitution par la proximité du casino, par l'attente d'un ami, ou encore par la curiosité, l'habitué des lieux se définissant comme non-client accepte de livrer «ce qu'il a entendu dire ». Le récit et les impressions sont alors souvent tellement circonstanciés qu'il est peu probable qu'ils n'aient été transmis que par ouï-dire, et l'habitué quant à lui, percevant que son discours pourrait être celui d'un client, se sent tenu de rappeler régulièrement qu'il ne livre là que des informations de seconde main. Il est possible qu'il soit lui-même conscient de la capacité de discernement de l'enquêteur, mais peu importe finalement la force probante de cette affirmation: son énonciation établit la coupure symbolique nécessaire entre deux statuts, celui de client et de non-client, rituel indispensable à certains avant tout échange relatif à la sexualité prostitutionnelle.

D'autres clients ne font, quant à eux, aucun mystère de leurs habitudes sexuelles mais cherchent au contraire à leur conférer une dimension « éthique ». C'est le cas notamment des hommes interrogés qui déclarent mettre un point d'honneur à n'avoir des relations sexuelles qu'avec des prostituées «volontaires » et « prenant plaisir à l'acte sexuel ». Alors même que la plupart des clients avouent par ailleurs leur incapacité à distinguer les prostituées travaillant sous la contrainte des prostituées dites «libres » (30) et pensent que la motivation des femmes se prostituant est avant tout financière, le plaisir de la prostituée est souvent invoqué par les clients. Pareille évocation situe en effet dans une démarche altruiste un comportement sexuel généralement considéré comme égoïste et vise ainsi à le rapprocher des standards de la sexualité traditionnelle, qui supposent la recherche d'un plaisir mutuel. Plus généralement, les clients interrogés se disent souvent sensibles, dans le choix des établissements qu'ils fréquentent, aux « ambiances », et en particulier au rôle du glamour et de la séduction. Outre le plaisir physique, certains clients recherchent donc également un contact ou une relation. Cette quête d'une reproduction des formes classiques de la relation amoureuse (Brochier, 2005 ; Hoigard et Finstad, 1992) constitue d'ailleurs un des motifs du recours à la prostitution transfrontalière : les femmes d'Europe centrale et orientale sont souvent décrites comme plus chaleureuses et moins arrogantes que les Européennes de l'Ouest car « pas encore atteintes par les valeurs d'émancipation féminine occidentales » (31). Le rôle, plus ou moins important selon les clubs, de la sensualité et de la séduction dans l'établissement de la relation prostituée/client, en replaçant la relation prostitutionnelle dans le contexte plus général des rapports amoureux et sexuels entre hommes et femmes, rassure le client sur sa capacité à séduire et à procurer du plaisir. La prostituée est alors décrite comme une partenaire sexuelle exceptionnelle, une "professionnelle du plaisir » capable de raviver une sexualité considérée jusqu'alors comme peu satisfaisante (Velten, 1994, p. 165). Certains clients reconnaissent ainsi des compétences particulières à la prostituée dans le jeu de la séduction

(30) Nous insistons sur le caractère nécessairement subjectif des notions telles que « consentement », « prostitution volontaire » ou «prostitution forcée » et sur l'usage précau- tionneux qui nous semble devoir en être fait.

(31) Citation d'un client à la frontière tchéco-autrichienne. 
et de l'amour, tel ce client interrogé à la frontière tchéco-autrichienne qui, expliquant son recours à la prostitution alors qu'il est marié, s'exclame : « Ici, les femmes sont tellement chaudes, on est à fond dedans ! » Ces hommes semblent alors rechercher dans le rapport prostitutionnel la possibilité de laisser l'entière initiative à leur partenaire, d'être « délestés du poids de celui qui doit toujours être le plus fort»(Mossuz-Lavau, 2005). La relation de pouvoir à l'œuvre dans le rapport prostitutionnel serait dans ce cas davantage marquée par cette « quête incertaine » (Howe, 2004) du client que par la position de pouvoir, souvent prédonnée, de l'homme sur la femme.

Cette représentation de la femme prostituée comme «professionnelle du plaisir», et bien sûr le moindre prix des services sexuels en République tchèque, motivent d'ailleurs également le recours des plus jeunes à la sexualité prostitutionnelle : les Allemands et Autrichiens entre 18 et 25 ans sont en effet nombreux à se rendre dans les lieux de prostitution transfrontaliers, qui conserveraient ainsi leur ancienne fonction d'initiation à la sexualité. Dans ce cas, le rôle de la séduction et du glamour semble cependant moindre que chez les clients plus âgés : certains clubs ont même mis en place des services spéciaux pour les clients plus jeunes, qui «veulent que ça aille vite » (32), tandis que les clients plus âgés sont plus nombreux à déclarer choisir les prostituées qu'ils pourraient imaginer avoir comme petites amies (Grenz, 2005) ou avoir déjà eu une relation amoureuse avec une prostituée. Le fantasme de l'établissement d'une relation amoureuse avec une prostituée experte du jeu de l'amour reste donc très ancré dans l'imaginaire des clients et témoigne d'une quête tant sentimentale que sexuelle.

La relation prostitutionnelle peut alors être comprise comme un vecteur de réaffirmation de la virilité du client, par ailleurs mise à mal par l'omniprésence de sollicitations sexuelles de plus en plus violentes dans l'espace public et médiatique, qui posent en « référence unique et indépassable [...] le coït hétérosexuel conjugal » (Mathieu, 1998), ainsi que par la stigmatisation dominante du recours aux services des prostituées comme le signe d'une incapacité à séduire.

Dans ce contexte, nous voyons dans les tentatives d'hérö̈sation par les clients du recours au sexe prostitutionnel une forme de réponse, voire de résistance, au stigmate du «déviant» (perdant ou pervers) associé au client de la prostitution. Les hommes interrogés, et ne se cachant pas de leur recours aux services des prostituées, mettent ainsi souvent en avant l'esprit curieux et aventurier dont témoigne leur envie de «voir à quoi ressemble » un rapport sexuel commercialisé. La dimension aventurière du recours au sexe prostitutionnel est par ailleurs fréquemment accentuée par l'invocation (en partie mythifiée) des risques inhérents à la fréquentation du milieu prostitutionnel, souvent décrit par les hommes interrogés comme «dangereux». Dans cet univers présenté comme régi par la seule loi criminelle, certains clients relatent avec fierté le rôle actif qu'ils ont déjà joué dans le «sauvetage » d'une

(32) Citation d'une prostituée travaillant en clubs à la frontière tchéco-allemande. 
prostituée contrainte et/ou violentée. Ces récits, dont la véracité importe peu pour notre propos, ne sont pas sans rappeler certaines formes classiques de narration héroïque d'inspiration chevaleresque, qui ont durablement influencé les représentations sociales des rôles sexués, et notamment la perception de certains comportements comme virils. En recourant au registre de l'aventure et de l'héroïsme, les clients mettent en exergue leur capacité d'homme à sauver une femme tout en se posant en défenseurs d'une prostitution « éthique ». Il va de soi qu'ils s'appuient pour ce faire sur une représentation de la prostituée contrainte qui leur est propre et en fonction de laquelle ils jugent de la nécessité ou non de se porter au secours des femmes qu'ils rencontrent, selon que celles-ci correspondent plus ou moins à l'image, forcément stéréotypée, qu'ils ont de la «victime ».

Or les images que les clients se font de leurs partenaires sexuelles prostituées dans les régions frontalières de l'ouest et du sud de la République tchèque sont selon toute apparence marquées par la perception de la présence des femmes d'Europe de l'Est dans la prostitution tchèque, mais aussi plus largement européenne, comme le signe de leur prédisposition, pour les formes commerciales de sexualité. Cette vision stéréotypique se trouve d'ailleurs confortée et alimentée par les estimations concernant la part croissante des prostituées d'Europe de l'Est sur les marchés du sexe ouest-européens (Bruinsma et Meershoek, 1997) comme par l'engouement des médias pour les portraits de femmes est-européennes se prostituant à l'Ouest (33). La plupart des clients que nous avons pu interroger insistent ainsi sur le caractère entièrement libre de l'exercice de la prostitution par les femmes d'Europe de l'Est dans les clubs de la frontière tchèque et sur leurs motivations exclusivement financières, et un consommateur de services sexuels à la frontière tchéco-autrichienne déclare par exemple : «Les femmes de l'Est sont intéressées par l'argent, c'est pour ça qu'on les trouve dans les bordels du monde entier. » Paradoxalement, les mêmes clients invoquant le plaisir de la prostituée et la dimension sensuelle et glamoureuse du contact comme facteurs motivant leur recours à la prostitution transfrontalière se retranchent derrière l'argument du caractère financièrement intéressé de l'entrée de ces femmes dans la prostitution dès lors que l'on aborde la question des éventuelles contraintes pesant sur les prostituées, migrantes notamment. Le choix du «business » prostitutionnel résulterait donc de l'attrait particulier exercé sur certaines femmes, est-européennes notamment, par la perspective de gains financiers. Cette affirmation par les clients des motivations matérielles de la prostituée, parce qu'elle ébranle le fragile édifice des tentatives de réinscription de la sexualité prostitutionnelle dans les standards de la relation amoureuse, est généralement servie par un discours beaucoup plus dur et désenchanté à l'égard de la prostituée : tant que l'aspect financier du rapport

(33) Voir notamment les nombreux articles relatant le «destin tragique » des « Natacha» contraintes à la prostitution en Europe de l'Ouest. Par exemple François Loncle, «L'Europe de l'Ouest, proxénète des femmes de l'Est»,
Le Monde diplomatique, novembre 2001 ; Florian Klenk, «Prostitution: der moderne Sklavenmarkt. Erkundungen im deutsch-tschechischen Grenzgebiet », Die Zeit, 40, 28/09/2006. 
n'est pas abordé, il semble que certains clients cherchent à présenter le contact avec la prostituée comme un rapport classique de séduction, où la beauté de la partenaire, sa capacité à séduire et l'ambiance de la rencontre déterminent l'issue de l'entreprise. Au contraire, dès qu'est rappelée la dimension pécuniaire du rapport, les discours des clients peuvent rapidement glisser dans un registre accusateur à l'encontre des prostituées, dont les considérations ostensiblement matérielles viennent contrarier les aspirations des consommateurs à une forme de sexualité perçue comme "normale». En choisissant de vendre leurs services sexuels pour en tirer un profit financier, les femmes se prostituant rappellent au client l'impossibilité d'un rapport amoureux désintéressé et sont alors accusées de n'être «pas des anges non plus» (34), et d'avoir donc «cherché » les ennuis éventuels qui peuvent accompagner l'exercice de la prostitution.

L'analyse des pratiques et des discours prostitutionnels semble donc révéler une perception majoritairement négative de leur sexualité par les clients, et les tentatives de justification d'un comportement perçu comme déviant qu'ils mettent en œuvre témoignent non d'une référence à une « sousculture déviante » (Sykes et Matza, 1957) qui présenterait le recours à la prostitution comme moralement correct, mais d'une demande de conformité avec les normes sociales faisant autorité. Bien que les clients de la prostitution frontalière en République tchèque se distinguent à bien des égards des délinquants juvéniles évoqués par Sykes et Matza, l'étude par ces auteurs des « techniques de neutralisation » de la désapprobation inhérente à l'internalisation de normes majoritaires paraît pouvoir éclairer certaines des formes de dédouanement discursif développées par les clients. Ainsi, le refus du client de concéder son recours à la sexualité prostitutionnelle, même si celui-ci est par ailleurs avéré, peut être analysé comme une technique de déni de la responsabilité (deny of responsability) destinée à briser le lien entre un individu et ses actes, tandis que l'invocation par le client de son engagement dans une forme de prostitution «éthique », en niant la potentialité d'une offense (deny of injury) pour les tiers (en l'occurrence, pour les prostituées), revient à nier toute corrélation entre un acte et ses éventuelles conséquences. Par ailleurs, sans pour autant retenir une approche de la prostitution en termes de victimes/coupables, l'on peut rapprocher les stratégies de neutralisation des clients par la mise en accusation des prostituées, présentées comme mues par l'appât du gain et taxées d'immoralité, des formes de "déni de la victime » (deny of the victim) observées par Sykes et Matza chez les délinquants. Cette dernière forme de rationalisation est notamment activée par les clients dans le cas des prostituées migrantes, dont la démarche migratoire à des fins de prostitution est généralement interprétée comme un signe de leur « culpabilité ».

(34) Citation d'un client interrogé à la frontière tchéco-autrichienne. 


\section{Les prostituées, victimes ou complices?}

Est-ce à dire que l'affirmation par la prostituée migrante de sa démarche volontariste d'entrée dans la prostitution dérange ? Le sens commun tend en effet à poser la migration prostitutionnelle d'Est en Ouest comme une démarche involontaire effectuée sous la contrainte (Geisler, 2005 ; Schauer, 2006) et à présenter les prostituées migrantes comme «des victimes réduites en esclavage, violées ou kidnappées »(Pheterson, 2003), quelles que soient les conditions réelles de leur entreprise migratoire. Au contraire, l'affirmation par la victime de son « consentement » initial à l'exercice d'une activité prostitutionnelle à l'étranger, parce qu'elle est le signe d'une «puissance d'agir sexuelle » (Butler, 2004, p. 137), est interprétée comme un aveu de complicité (y compris chez les clients, pourtant eux-mêmes par ailleurs victimes du stigmate qui frappe les différents acteurs du milieu prostitutionnel). C'est donc l'autonomie sexuelle et migratoire qui semble constituer la transgression (Pheterson, 2003) au sens moral universel tel que nous le concevons, et qui conduit à ne considérer les prostituées qu'à travers les mécanismes sociaux de contrôle, de stigmatisation et de soumission (à l'égard des clients, des proxénètes, des policiers, etc.). C'est pourquoi il importe ici, sans jamais nier la réalité des menaces physiques et psychiques pouvant peser sur les femmes exerçant la prostitution, de rendre plus lisibles certains des rapports de pouvoir traversant l'espace prostitutionnel.

Comme le laissent entendre les remarques exposées ci-dessus sur le comportement sexuel des consommateurs de services prostitutionnels, les relations entre clients et prostituées n'ont pas toujours « la froideur impersonnelle et mécanique généralement décrite », mais peuvent au contraire s'inscrire dans «certaines formes de communication et d'échange » (Brochier, 2005). Celles-ci semblent pouvoir être rapprochées des observations recueillies par C. Brochier dans les clubs de Rio de Janeiro et indiquent la possibilité d'une relative marge de manœuvre pour les prostituées dans le commerce et la négociation de prestations sexuelles. Tout d'abord, contrairement au stéréotype de victime de la traite dont est souvent investie la prostituée migrante, il semble que la plupart des femmes étrangères se prostituant à la frontière tchèque envisagent la vente de services sexuels à des clients ouest-européens réputés fortunés comme une stratégie économique leur permettant de faire face à un besoin financier. La démarche est donc très souvent initialement volontaire (35) (ce qui n'exclut naturellement pas les cas de traite des femmes ou l'apparition de formes ultérieures de contraintes) et le fait de femmes qui savent quel genre de travail les attend, même si elles peuvent éventuellement avoir espéré d'autres conditions de travail (Šídová, 2004a). Les étrangères, pour la plupart originaires de l'ancien bloc socialiste, exerçant dans les clubs des régions frontalières se prostituent fréquemment pour résoudre temporairement une situation économique difficile et utilisent

(35) Entretien avec les représentants d'une ONG de prévention sanitaire auprès des prostituées à la frontière tchéco-bavaroise. 
pour ce faire différentes portes d'entrée dans la prostitution : si la plupart arrivent en République tchèque avec l'intention de travailler dans l'industrie du sexe munies d'un visa de tourisme de trois mois, la procédure d'asile ou encore le mariage avec un citoyen tchèque (ou éventuellement allemand ou autrichien) sont également utilisés comme outils de régularisation du séjour par les femmes venues travailler dans le commerce sexuel. Elles entrent généralement en contact avec des clubs par le biais d'un intermédiaire, qui prend également en charge l'obtention d'un visa de tourisme et l'organisation de leur voyage. Ces intermédiaires, proxénètes endossant parfois aussi le rôle de petit ami, disposent en Tchéquie d'un réseau de contacts parmi les propriétaires de clubs, auxquels les filles sont ensuite proposées. Après l'engagement de la prostituée migrante dans un club, l'intermédiaire conserve généralement son rôle de proxénète et prélève une partie des gains de la prostituée (36). Le contact avec le propriétaire du club peut cependant éventuellement être également établi par le biais d'une prostituée exerçant déjà dans un établissement et qui y organise l'embauche d'une amie ou d'une connaissance. Il n'est par ailleurs pas rare, dans le cas des femmes ukrainiennes notamment, que l'activité prostitutionnelle s'inscrive dans une perspective plus large de migration de main-d'œuvre depuis l'Ukraine vers la République tchèque : certaines femmes initialement venues travailler en usine ou dans la restauration, et dont les salaires dépassent alors à peine le minimum vital, trouvent un avantage financier à se lancer dans la prostitution en clubs ou en appartement (Š́́dová, 2004b). La plupart des femmes munies d'un visa de tourisme rentrent dans leur pays d'origine après trois mois d'activité afin de renouveler leur visa, puis reviennent en République tchèque (ou partent éventuellement plus à l'Ouest). Certaines des prostituées travaillant en clubs ont également un titre de séjour d'un an, obtenu suite à l'acquisition d'une licence commerciale (très facile à obtenir en République tchèque) et à leur enregistrement en tant qu' « entrepreneur».

Une part importante des femmes travaillant dans les clubs semble donc envisager ce passage par la prostitution en région frontalière soit comme une activité transitoire pour subvenir aux besoins de leur famille dans le pays d'origine, soit comme une étape dans leur carrière prostitutionnelle, qu'elles cherchent à poursuivre dans d'autres villes européennes où la vente de services sexuels est financièrement plus intéressante (37). Dans cette perspective, elles estiment souvent normal de payer un intermédiaire leur procurant une place dans un club (38). De la même façon, elles ne remettent apparemment en cause, tant qu'elles vivent dans les clubs, ni le fait de reverser une part importante de leur salaire au gérant, ni même l'exercice par le gérant de certaines formes de contrainte verbale et psychologique, considérées comme faisant « partie du métier » (39) (Hoigard et Finstad, 1992). Les conditions de

(36) Voir plus haut.

(37) Voir les entretiens conduits avec des femmes se prostituant en clubs à la frontière tchéco-allemande.

(38) Voir les informations recueillies auprès d'ONG tchèques et allemandes d'aide aux prostituées.

(39) Citation d'une prostituée à la frontière tchéco-allemande. 
travail que les observateurs extérieurs, pétris de sentiments protecteurs, tendraient à qualifier rapidement d'esclavagistes peuvent donc être envisagées sous un jour entièrement différent par les prostituées elles-mêmes, pour lesquelles l'entrée dans le milieu prostitutionnel paraît signifier l'acceptation des règles qui en régissent le fonctionnement. Ainsi, certaines femmes identifiées comme victimes de la traite en République tchèque choisissent malgré tout de poursuivre leur activité prostitutionnelle, sachant qu'elles peuvent gagner en quelques mois à la frontière tchèque assez d'argent pour vivre ou étudier plusieurs années en Ukraine par exemple (40). Encore une fois, il ne s'agit nullement de remettre en question ici la surexposition des femmes prostituées à différentes formes de violence, mais simplement de ne pas laisser certains a priori victimisants occulter la réalité de l'entreprise prostitutionnelle et sa possible perception par les prostituées elles-mêmes comme une forme d'échange entre les parties impliquées - le gérant du club, la prostituée et le client.

L'observation des termes de l'échange entre clients et prostituées dans les lieux de prostitution infirme par ailleurs les interprétations manichéennes de clients dominants exploitant les prostituées dominées et révèle au contraire, chez les prostituées, un «certain mode de contrôle sur la pénibilité de l'activité » (Brochier, 2005). Ce contrôle nous semble notamment passer par «l'apprentissage de l'intériorisation d'un stigmate et du moyen de le manipuler »(Pryen, 1999, p. 151) : face au stigmate qui frappe l'ensemble des prostituées, et peut-être plus encore les prostituées migrantes (davantage soumises aux pressions policières, mais aussi victimes de manifestations de rejet par les autres membres de la communauté prostitutionnelle, qui les accusent de contribuer à la stigmatisation de la profession) (41), la distanciation qu'elles pratiquent souvent à l'égard de certains aspects de leur activité et de leur statut prostitutionnel peut être analysée comme l'une des formes d'apprentissage et de manipulation de la stigmatisation. Outre la distanciation symbolique que le code vestimentaire réservé à la prostitution est censé établir entre deux statuts, «la figure paradigmatique de la "putain" et ellesmêmes »(Welzer-Lang, Barbosa et Mathieu, 1994, p. 149), la plupart des femmes prostituées tiennent visiblement à préserver une certaine distance avec le client, comme en témoignent certaines déclarations des clients remarquant que «ces filles sont très douées pour cacher ce qu'elles ont besoin de cacher » (42) : se livrer reviendrait en effet à brouiller cette coupure symbolique entre deux identités, qui donne le sentiment à la femme prostituée de conserver son intégrité dans la pratique prostitutionnelle (Guillemaut, 2002, p. 50). Par ailleurs, la distanciation à l'égard du client permet à la prostituée de conserver en toutes circonstances le contrôle de la situation : la prostituée «ne livre rien de sa vie privée. Il s'agit d'être en représentation, pour

(40) Entretien avec les membres d'une ONG de prévention sanitaire auprès des prostituées à la frontière tchéco-bavaroise.

(41) Les Ukrainiennes sont généralement accusées par les prostituées tchèques et slovaques d'accepter des conditions d'exercice de leur activité dégradantes, sans protection notamment.

(42) Citation d'un client à la frontière tchéco-allemande. 
satisfaire la demande du client, sans investir de soi. Cette mise en scène est reproductible dans toutes les relations aux clients, même si l'enjeu est parfois de faire "comme si" cette relation était unique» (Pryen, 2002). Les femmes prostituées interrogées affichent d'ailleurs souvent un certain mépris pour leurs clients dupes de cette mise en scène, dont elles commentent sans complaisance les performances sexuelles. Le vocabulaire utilisé par les prostituées pour parler des clients sert également à signifier cette coupure avec les touristes sexuels étrangers, parfois désignés par le terme «prasátka» (cochons), notamment lorsqu'il leur est reproché de ne pas respecter les règles de protection des rapports sexuels (43). Les rares clients vietnamiens de la prostitution frontalière sont particulièrement exposés au mépris des prostituées: alors même que la population locale se montre généralement prompte à amalgamer la présence d'une importante communauté vietnamienne et l'existence de nombreux lieux de prostitution à la frontière, les clients vietnamiens sont unanimement rejetés par les prostituées interrogées, qui les considèrent comme physiquement peu attrayants et sexuellement peu performants. Les entretiens conduits avec les prostituées et les observations effectuées au sein de certains clubs infirment d'ailleurs l'idée que les femmes sont tenues d'accepter tous les clients se présentant. Les prostituées déclarent généralement ellesmêmes ne pas avoir d'obligation d'accepter un client (si ce n'est qu'elles doivent pouvoir être en mesure de payer la location de leur chambre à la fin du mois) et, même si aucun des clients interrogés n'a reconnu avoir déjà été éconduit par une prostituée, les situations que nous avons pu observer dans les clubs témoignent de la possibilité de ce cas de figure. Certains lieux de petite taille peuvent en effet donner le sentiment à l'observateur extérieur, mais également au client potentiel qui y pénètre, de troubler la quiétude du salon familial : quelques filles y sont allongées sur le canapé devant la télévision, et les rares clients qui poussent la porte du club les soirs de semaine sont souvent jugés moins dignes d'intérêt que les derniers clips vidéos et donc abandonnés au comptoir, jusqu'à ce qu'ils décident d'eux-mêmes de partir. Ces clubs sont d'ailleurs la cible privilégiée des opérations de marketing des établissements plus grands et plus luxueux, qui stigmatisent le manque d'ambiance des petites structures ne méritant pas à leurs yeux le qualificatif de «night club» (44). Sans prétendre que la configuration de ces « clubs-salons » soit représentative de l'ensemble de la prostitution frontalière en clubs, nous voyons là le signe d'une réalité prostitutionnelle très diverse et bien moins monochrome que l'image que l'on pourrait en avoir.

Par ailleurs, la capacité des prostituées à donner aux clients le sentiment que chaque rencontre est irreproductible, ainsi que l'aptitude qui leur est publiquement reconnue à contrôler le plaisir masculin, nous semblent également constituer, pour les prostituées, un vecteur important de manipulation et de réappropriation du stigmate de prostituée pouvant leur permettre de résister, ne serait-ce qu'en apparence, au «point de vue défavorable et

(43) Informations recueillies auprès des membres d'une ONG d'aide aux prostituées à la frontière tchéco-autrichienne.

(44) Voir plus haut. 
résigné » (Mathieu, 2000) qu'elles tendent à adopter sur elles-mêmes. La mise en scène de leur corps (par les vêtements, la «tenue » [45], l'exposition, etc.) comme outil de pouvoir sur le désir masculin, par la suggestion d' « une forme d'érotisme recherchée par le client »(Gil, 2005, p. 349), peut en effet conférer aux femmes se prostituant un sentiment de puissance, même temporaire : la confrontation avec d'autres femmes non issues de la communauté prostitutionnelle (en l'occurrence avec l'enquêtrice) paraît ainsi éveiller chez les femmes prostituées, indépendamment d'une certaine nostalgie résignée relative à leur glissement dans un groupe social stigmatisé (Goffman, 1975, p. 7), la conscience de leur capacité à incarner une forme de rapport libéré au corps et à la sexualité, dont elles peuvent jouer pour faire apparaître leurs interlocutrices non prostituées comme rigides et engoncées dans différents tabous sexuels. Ainsi, une prostituée interrogée en extérieur (à l'arrêt de bus du village), après s'être enquise du parcours professionnel et universitaire de l'enquêtrice, a d'abord déclaré, d'un air attristé, regretter d'avoir «atterri dans la prostitution » pour avoir préféré la « fête » à ses études. Pourtant, son choix, quelques instants plus tard, de se dévêtir et de poursuivre l'entretien en sous-vêtements au beau milieu d'un espace public peut être interprété comme une manière de signifier à l'enquêtrice qu'elle accède à d'autres formes de liberté que celle qu'est censée conférer l'occupation d'une position sociale non stigmatisée. Certes, il ne s'agit peut-être là que d'une apparente libération sexuelle, d'une mise en scène de libération sexuelle par des femmes dont le corps est avant tout un instrument d' " accumulation économique » (Mathieu, 2000), et la libération sexuelle ne saurait se mesurer à l'aune de la capacité des femmes à exposer et instrumentaliser leur corps. Il n'empêche que cette forme de théâtralisation du corps visant à le faire apparaître non seulement comme catalyseur du plaisir masculin mais aussi comme incarnation d'une sexualité sans tabous nous semble pouvoir être analysée, avec les tentatives de distanciation présentées ci-dessus, comme une des formes de résistance mises en œuvre par les femmes prostituées pour échapper au regard misérabiliste que l'on incline à porter sur elles.

La survictimisation des prostituées conduit également à méconnaître les capacités de discernement dont elles ont à faire preuve dans l'exercice de leurs activités afin d'éviter les situations dangereuses, les clients potentiellement pervers et les risques sanitaires inhérents à la vente de services sexuels. Les prostituées interrogées à la frontière tchéco-allemande présentent en effet les clients à tendance sadomasochiste et les maladies sexuellement transmissibles comme les principaux dangers de l'activité prostitutionnelle, requérant une vigilance de chaque instant et une certaine capacité à décrypter les schèmes comportementaux du consommateur de services sexuels (Gil, 2005). Bien que la plupart des clients rencontrés dans le cadre de cette étude s'en défendent, ils seraient encore nombreux à proposer à leur partenaire prostituée un supplément de prix pour un rapport non protégé et à tenter de

(45) Au sens que Goffman donne à ce terme, à savoir «le maintien, le vêtement, l'allure et qui sert à montrer à l'entourage que l'on est une personne douée de certaines qualités favorables ou défavorables » (1974, p. 69). 
tromper la vigilance des prostituées imposant l'usage du préservatif (46). Les prostituées insistent également sur les dangers que leur font encourir les fantaisies sexuelles de certains clients, et qu'il importe de déceler à temps pour s'y soustraire. Ainsi, ayant constaté une propension supérieure des clients plus âgés à développer des tendances sadomasochistes dans leurs rapports, certaines prostituées ont déclaré se protéger en refusant systématiquement les clients paraissant plus de soixante ans. Cette affirmation, tout comme l'observation des pratiques prostitutionnelles au sein des clubs, témoigne de l'existence possible, pour les prostituées, d'une marge de manœuvre leur permettant de refuser certains clients. Par ailleurs, la solidarité entre prostituées, généralement décrite comme déclinante dans les lieux de prostitution traditionnels (Mathieu, 2000), paraît exister à certains moments et sous certaines formes précises dans les nouvelles régions prostitutionnelles que sont les espaces frontaliers de la République tchèque : les prostituées travaillant en clubs à la frontière tchéco-allemande expliquent ainsi relever systématiquement les numéros de plaque d'immatriculation des clients avec lesquels les filles partent parfois pour des services d'escorte afin de pouvoir avertir la police si une collègue tardait à reparaître.

Le confort qu'offre la représentation de la prostituée comme victime et du client comme pervers, en permettant à l'observateur de se distancier de figures emblématiques qui le mettent mal à l'aise et auxquelles il s'attache à ne pouvoir s'identifier, entrave le décloisonnement de cette zone confinée qu'est la prostitution frontalière et l'analyse des motivations qui la traversent. Il nous semble toutefois que les tentatives pour se dégager des figures stéréotypiques du client et de la prostituée, et des prénotions qu'elles véhiculent, conduisent à reconnaître la possibilité que certaines femmes décident de travailler dans l'industrie du sexe comme circuit alternatif de survie (tout en gardant à l'esprit que les aspects de la pratique prostitutionnelle observés ici ne sauraient rendre compte de l'ensemble de la réalité prostitutionnelle), et que le rapport entre prostituée et client ne soit pas qu'un simple rapport de domination, mais puisse prendre la forme d'un échange. Pourtant, la formulation de l'hypothèse d'une recherche de «coopération avantageuse » (Brochier, 2005) entre les parties impliquées dans le milieu prostitutionnel est généralement accusée, dans un débat européen sclérosé par l'opposition entre réglementaristes (47) et abolitionnistes (48), de nier la réalité des violences

(46) Voir les déclarations des prostituées et des représentants d'organisations d'aide aux prostituées.

(47) Les réglementaristes, considérant que la prostitution est un fait social inévitable, voire nécessaire, préconisent de la contrôler en la soumettant à certaines règles (quartiers réservés, maisons closes ou eros-centers, registres et fichiers de police, surveillance médicale des personnes prostituées, etc.). Institué en France en 1802, ce régime (dénommé « Système français ») a persisté jusqu'en 1946. Il est soutenu en
Europe par certains pays, souvent de culture germanique, et notamment les Pays-Bas, l'Allemagne, l'Autriche, la Suisse, mais aussi la Grèce et la Turquie.

(48) La position abolitionniste voit dans la prostitution une forme d'institutionnalisation de la «domination masculine » qu'il convient de prévenir et de combattre. La France, l'Italie, la Belgique et la Grande-Bretagne sont officiellement de conception abolitionniste (bien que les positions abolitionnistes de certains se teintent parfois de réglementarisme). 
faites aux femmes prostituées. Il ne s'agit pourtant aucunement de remettre en question la surexposition des prostituées à diverses formes de contraintes physiques et psychologiques mais de chercher à redonner à chacun des acteurs sa place dans la sociologie du milieu prostitutionnel.

\section{Frontière, déviance et stigmatisation}

En raison de la surreprésentation des prostituées étrangères et des clients germanophones dans le milieu prostitutionnel frontalier, celui-ci est majoritairement perçu par les acteurs locaux comme le point de convergence d'intérêts étrangers à la République tchèque. Il semble en fait que, autour du marché du sexe frontalier, se cristallise la pensée de l'Autre comme moralement et culturellement différent. Ainsi, les femmes tchèques et plus généralement «est-européennes » se prostituant sont généralement perçues par les hommes interrogés comme plus disponibles pour le commerce sexuel que les femmes « occidentales » car incarnant « un rapport différent au sexe et à l'argent» (49). Cette perception, que nous avons eu l'occasion d'évoquer plus haut, repose plus ou moins implicitement sur la notion de «civilisation » (O'Connell Davidson, 2001), dont l'état d'avancement conditionnerait le rapport des femmes à l'acte sexuel monnayé : les valeurs d'émancipation féminine n'auraient pas encore atteint les pays situés à l'est de l'Europe (50), et ce «retard civilisationnel» expliquerait la plus forte propension des femmes à monnayer leurs services sexuels. Parce qu'elles incarnent par ailleurs sans détours le rapport sexuel monnayé aux yeux de la population locale, c'est-à-dire le sexe qui dérange en ce qu'il ne correspond alors plus à l'image de communion physique et psychique entre deux êtres que l'on incline à s'en faire, et qu'elles sont étrangères aux clients comme aux locaux (51), les prostituées migrantes transgressent une double frontière - celle du sexe traditionnel et celle de la souveraineté nationale (Berman, 2003).

Deuxième figure de l'Autre dans les représentations de l'espace prostitutionnel, le client transgresse finalement les mêmes frontières, nationales et sexuelles, que les prostituées migrantes et est donc frappé par le même stigmate de déviance morale et sexuelle: pour le non-client, le client est le «perdant» des régions frontalières allemandes et autrichiennes qui, ne pouvant prétendre à une vie amoureuse et sexuelle «normale », passe la frontière pour assouvir ses envies plus ou moins perverses dans les clubs de la République tchèque. Le franchissement de la frontière nationale paraît alors

(49) Citation d'un client à la frontière tchéco-autrichienne.

(50) Déclarations recueillies auprès de clients à la frontière tchéco-autrichienne.

(51) Outre les prostituées migrantes originaires d'anciens États socialistes, les prosti- tuées tchèques ne sont généralement pas issues des régions dans lesquelles elles exercent et les femmes roms se prostituant dans la rue sont perçues comme étrangères et taxées d'immoralité. 
investi d'un pouvoir, ou tout au moins d'une promesse, d'émancipation sexuelle (Donnan et Wilson, 1999, p. 91), permettant la réalisation de formes de sexualité considérées comme impossibles sur le territoire du pays d'origine.

Mais ce stigmate moral et sexuel (voire éventuellement criminel) qui entoure clients et prostituées semble s'appliquer également, dans le paysage frontalier tchèque, à d'autres figures de l'altérité qui, si elles ne constituent pas directement l'objet de notre propos, ont pu contribuer à la représentation sociale de l'espace frontalier comme régi par des intérêts extérieurs. D'une part, la surexposition des prostituées roms dans la prostitution de rue (52) et leur perception par les populations locales comme immorales et liées à différents réseaux criminels ont potentiellement influencé certaines représentations de la prostitution frontalière, en clubs comme de rue. En effet, leur image de femmes de peu de vertu jetées sur le trottoir par un mari, un frère ou un père violent et criminel, semble avoir favorisé dans la population locale une forme d'amalgame entre Roms et prostitution (53), et plus largement entre prostitution et criminalité, qui encourage la perception de la prostituée comme étrangère et exclue des valeurs morales dominantes (54). D'autre part, la présence d'une importante communauté vietnamienne, qui s'est s'établie dans les régions frontalières de la République tchèque depuis la chute des régimes communistes et l'ouverture des frontières, pourrait avoir, bien qu'elle ne constitue pas directement un acteur du milieu prostitutionnel, facilité certaines associations entre «étranger» et « désordre» dans les imaginaires locaux. En effet, les Vietnamiens, venus en Tchécoslovaquie et dans la RDA voisine dès les années 1960 à la faveur de contrats de travail conclus avec la République socialiste du Vietnam, se sont installés dans les régions frontalières de la République tchèque au début des années 1990, après que les changements politiques survenus dans les anciens États socialistes avaient rendu caducs les accords en vertu desquels ils étaient jusqu'alors employés dans les usines tchécoslovaques et est-allemandes. Ils se sont alors lancés dans le négoce de produits de fabrication asiatique et sont à l'origine de l'organisation, à la frontière tchéco-allemande puis tchéco-autrichienne, d'immenses «bazars » spécialisés dans le commerce d'articles de contrefaçon, de cigarettes et de nains de jardin. Or, bien qu'aucun lien n'existe a priori entre les marchés du sexe et les activités de la communauté vietnamienne, celle-ci est souvent évoquée, par les habitants tchèques comme par les clients ou touristes germanophones, dès qu'est abordée la question de la prostitution frontalière. Cet amalgame sans fondement objectif pourrait être, entre autres, imputé au fait que, comme pour les établissements de prostitution, l'existence des

(52) La prostitution sur les routes de la plupart des régions frontalières est majoritairement le fait de femmes roms.

(53) Perception encouragée par la médiatisation des opérations de police visant les proxénètes roms. Voir par exemple le démantèlement par la Brigade de répression du crime organisé, en septembre 2006, d'un groupe de proxénètes trafiquants décrit comme l'un des plus importants en République tchèque et dont les principaux membres étaient issus de familles roms.

(54) Même si la plupart des prostituées roms dans les régions frontalières ont la nationalité tchèque, ou éventuellement slovaque. 
marchés vietnamiens repose sur le différentiel économique important existant entre la République tchèque et ses voisins « ouest-européens ». Outre l'image de communauté fermée et peu intégrée généralement associée à la population vietnamienne installée en République tchèque (Hurrle, 2006), qui paraît avoir encouragé sa perception comme secrète et potentiellement criminelle (55), il est souvent reproché aux Vietnamiens des zones frontalières (tout comme aux établissements de prostitution) d'avoir terni, par l'installation d'immenses marchés de plein air jugés inesthétiques, l'image de ces régions et d'attirer une forme de tourisme «discount» et peu glamour.

Vietnamiens et Roms semblent ainsi se retrouver eux aussi à l'intersection de deux formes de stigmatisation, les mêmes qui frappent clients et prostituées et qui, alliant stéréotypes racistes et jugements moraux, voient dans l'étranger à la fois un facteur de perversion de l'image et des mœurs de la région, et un agent au service de réseaux criminels tirant profit d'activités peu avouables. Des initiatives de citoyens se sont d'ailleurs mises en place dans certaines communes frontalières tchèques, avec pour objectif $d$ ' " apporter des solutions aux problèmes de la prostitution, des Vietnamiens et des Roms », témoignant de l'imbrication de ces trois phénomènes dans l'imaginaire collectif des populations frontalières (56).

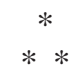

La prostitution frontalière, de par sa situation « aux confins » et le caractère majoritairement étranger des acteurs qu'elle implique ou que l'on associe communément, à tort ou à raison, aux activités prostitutionnelles, peut ainsi être vue comme l'archétype d'un espace séparé, d'une zone frontière «en rupture avec l'extérieur » (Brochier, 2005). Le degré très élevé de concentration et de commercialisation de l'offre de services sexuels dans les régions frontalières de la République tchèque est généralement perçu par les populations locales comme le fruit de la rencontre, à la frontière, des immoralités étrangères. Les marchés du sexe des espaces frontaliers seraient alors en quelque sorte le «prix à payer » pour l'ouverture des frontières de la Tchéquie, conduisant à la perception de la proximité des frontières allemande et autrichienne comme, simultanément, «l'un des principaux atouts [...] et l'une des plus grandes faiblesses » des régions frontalières tchèques (57). Cette représentation a pour corollaire une relative tabouisation de la suractivité prostitutionnelle frontalière (58), dont l'existence même est vécue

(55) Représentation confirmée par les entretiens conduits avec des habitants des régions frontalières de la République tchèque.

(56) Rendlová Z., 2004. - « Das Bild des deutsch-tschechischen Grenzraums in der tschechischen Zeitung "Chebský deník" im letzten Quartal 2003 », Faculté de Philosophie de l'Université technique de Chemnitz. Disponible sur http://www.tu-chemnitz.de/phil/europastudien/download/fond-cz/ws0304_bericht- rendlova.pdf (16/08/2006).

(57) Maire de Cheb, ville située à la frontière tchéco-allemande, cité dans Hurrle (2006, p. 21).

(58) Voir par exemple le court métrage «České Velenice Infinity » dans le film documentaire Across the border. Five views from neighbours, par Pawel Loziński, Jan Gogola, Peter Kerekes, Robert Lakatos and Biljana Čakič-Veselič, Autriche, 2004. 
comme une remise en cause de l'image de progrès social habituellement associée à la sortie du communisme. Dans cette perspective, la République tchèque aurait dû accepter, ou plutôt feindre d'ignorer, la transformation de ses frontières du Nord, de l'Ouest et du Sud en immenses marchés du sexe à destination des touristes européens (et notamment allemands et autrichiens) en échange justement de son «retour à l'Europe». Ainsi, malgré les initiatives des communes des régions frontalières tchèques en faveur d'une réglementation de la prostitution (59), le rapport au commerce du sexe semble d'une certaine manière se «normaliser » dans les espaces frontaliers. La visibilité des prostituées dans les espaces publics, et le fait par exemple qu'elles fréquentent souvent les mêmes cafés ou les mêmes magasins que les locaux (qui, interrogés personnellement, déclarent généralement entretenir de bons rapports avec les femmes se prostituant) témoignent, par exemple, d'une possible dédramatisation de la sexualité prostitutionnelle. Certes, comme nous l'avons montré, l'installation massive d'établissements de prostitution est accusée de ternir l'image des régions frontalières, et les prostituées sont partie prenante de ce processus, mais les rapports individuels des habitants avec les femmes engagées dans le commerce du sexe paraissent malgré tout partiellement libérés de certaines formes de stigmatisation : on les salue dans la rue, on s'enquiert de leur santé, et un aquarelliste, marié et retraité et dont la maison est entourée de deux clubs, déclare prendre parfois les prostituées comme modèles pour ses portraits. On remarque par ailleurs, depuis quelques années, une présence accrue d'hommes tchèques dans les établissements de prostitution frontaliers. Les clubs deviennent notamment, pour un nombre croissant de jeunes Tchèques habitant les régions frontalières, des lieux de sortie nocturne au même titre que les bars plus «classiques », sans que leur soit forcément associée la consommation de services sexuels (60). De manière générale, et bien qu'elle reste stigmatisée, l'activité prostitutionnelle paraît comparativement bénéficier d'une plus grande «acceptabilité » morale dans les zones frontalières tchèques que dans l'Allemagne ou l'Autriche voisines, où la prostitution est pourtant reconnue comme profession. Le développement de la prostitution comme fait social «acceptable » dans les États post-socialistes a de fait souvent été présenté comme le résultat de la chute des régimes communistes et de l'indépendance nationale, lesquelles auraient favorisé l'apparition d'une «icône pornographique » (Watson, 1993) comme nouveau symbole de liberté. Réprimée sous le régime communiste, la prostitution et sa reconnaissance comme phénomène de société ont en effet pu apparaître, au cours des années 1990, comme le signe d'une libération sexuelle. Par ailleurs, le contexte économique et politique des années 1990, marqué notamment par une fragilisation des femmes sur le marché du travail

(59) Les représentants de 25 communes ont par exemple adopté une déclaration en août 2000 appelant le gouvernement et le parlement tchèques à agir contre la prostitution dans les régions frontalières. Certaines communes frontalières ont également pris des initiatives individuelles, telle la ville de Aš, qui a introduit en février 2006 des panneaux signalant les emplacements auxquels la prostitution est autorisée et ceux où elle est interdite.

(60) Hommes tchèques interrogés à la sortie d'un club à la frontière tchéco-allemande. 
ainsi que par l'accroissement des libertés individuelles et l'ouverture des frontières du pays, aurait pu contribuer au développement d'une forme de «banalisation » de la prostitution associée à certains territoires « en marge », et en particulier aux régions frontalières. Les prostituées semblent ainsi pouvoir y jouir d'une tolérance de fait, possiblement motivée par certaines considérations économiques sur la place des femmes, et plus encore de la femme migrante, sur le marché du travail. Au contraire, la plupart des Allemands et des Autrichiens interrogés dans les régions frontalières tchèques déclaraient avec véhémence que pareille intégration des clubs de prostitution dans le paysage frontalier allemand ou autrichien serait « impensable ». Fautil voir dans cette apparente dédramatisation - du côté tchèque - du sexe commercialisé le signe d'un glissement progressif des termes de la «division morale du travail » (Hughes, 1996, p. 63) dans les zones frontalières, qui résulterait à la fois de la prise de conscience des contraintes économiques pesant sur les femmes se prostituant et de la présence d'un réservoir important et directement accessible de clients potentiels ? La frontière, de par son caractère franchissable, serait alors à la fois l'institution rendant possible la sexualité prostitutionnelle frontalière et l'instrument de réaffirmation d'une limite entre acceptable et inacceptable, par un processus de répartition des différentes fonctions morales (ibid., p. 100) de part et d'autre des frontières nationales. Bien qu'ouverte, la frontière conserverait alors pour les pays ouesteuropéens voisins une part de son ancienne matérialité, permettant la relégation du «sale boulot», allant à l'encontre de «nos conceptions morales les plus héroïques» (ibid., p. 81), de l'autre côté des limites nationales, en l'occurrence en République tchèque. Ce déplacement de l'acceptable aux abords des frontières nationales serait cependant également ce qui distingue l'espace confiné de la prostitution frontalière des formes de sexualité prostitutionnelle sur le reste du territoire tchèque. De par sa situation « en marge », la prostitution frontalière pourrait ainsi offrir un terrain privilégié pour comprendre la charge affective et fantasmatique dont sont investis les lieux de prostitution (Botelho de Mattos et Angelo Campos Ribeiro, 1997), et leur rôle dans les représentations individuelles et collectives.

Mathilde DARLEY

IEP de Paris

Centre Français de Recherche en Sciences Sociales (CEFRES)

Vyšehradská 49

12800 Prague 2 - République tchèque

mathilde@cefres.cz.

\section{RÉFÉRENCES BIBLIOGRAPHIQUES}

Berman J., 2003. - «(Un)popular strangers and crises (un)bounded : discourses of sex-trafficking, the European political community and the panicked state of the modern state », European journal of international relations, 9, 1, pp. 37-86.

Bishop R., Robinson S. L., 1998. - Night market : sexual cultures and the Thai economic miracle, London, Routledge. 
Botelho de Mattos R., Angelo Campos Ribeiro M., 1997. - « Les territoires de la prostitution dans les espaces publics de la zone centrale de Rio de Janeiro », Géographie et cultures, 24, pp. 9-28.

Bouamama S., 2004. - L'homme en question. Le processus du devenir-client de la prostitution, IFAR, Mouvement du Nid, disponible sur: http://www.mouvementdunid.org/IMG/pdf/HommeEnQuestion.pdf (18/08/2006).

Brochier C., 2005. - «Le travail des prostituées à Rio de Janeiro », Revue française de sociologie, 46, 1, pp. 75-113.

Bruinsma G. J. N., Meershoek G., 1997. - «Organized crime and trafficking in women from Eastern Europe in the Netherlands », Transnational organized crime, 3, 4, pp. 105-119.

Butler J., 2004. - Le pouvoir des mots. Politique du performatif, Paris, Éditions Amsterdam.

Corbin A., 1978. - Les filles de noce. Misère sexuelle et prostitution aux $19^{e}$ et $20^{e}$ siècles, Paris, Éditions Aubier Montaigne.

Donnan H., Wilson T. M., 1999. - Borders. Frontiers of identity, nation and state, Oxford, Berg.

Geisler A., 2005. - Gehandelte Frauen. Menschenhandel zum Zweck der Prostitution mit Frauen aus Osteuropa, Berlin, Trafo-Verlag.

Gil F., 2005. - «Sexualité et prostitution» dans M.-É. Handman, J. Mossuz-Lavau (dirs.), La prostitution à Paris, Paris, Éditions de la Martinière, pp. 345-376.

Goffman E., 1974. - Les rites d'interaction, Paris, Éditions de Minuit.

- 1975. - Stigmate. Les usages sociaux des handicaps, Paris, Éditions de Minuit.

Grenz S., 2005. - « Sex tourism and trafficking. The perspective of male heterosexual clients of prostitutes », Conference Women's World 05, Seoul (Korea), University Ewha, June.

Guillemaut F., 2002. - Femmes et migrations. Les femmes venant d'Europe de l'Est, Lyon, Éditions Cabiria.

Hoigard C., Finstad L., 1992. - Backstreets, prostitution, money and love, Cambridge, Polity Press.

Howe Ch., 2004. - «Ergebnisse der qualitativen Studie über Kunden von ausländischen Prostituierten », Actes du colloque Prostitutionskunden. Sich austauschen, um Standpunkte zu verrücken, context e.V., pp. 31-45.

- 2006. - «Bilderwelten-Innenwelten. Prostitution und das Verhältnis der Geschlechter», Osteuropa, 6, pp. 67-85.

Hughes E. C., 1996. - Le regard sociologique. Essais choisis, Paris, Éditions de l'EHESS.

Hurrle J., 2006. - « Město a tržiště », Kulturní Týdeník, 10, p. 21.

Månsson S.-A., 2006. - « Men's demand for prostitutes », Sexologies, 15, 2, pp. 87-92.

Mathieu L., 1998. - «Le fantasme de la prostituée dans le désir masculin », Panoramiques, 34, pp. 72-79.

- 2000. - «L'espace de la prostitution. Éléments empiriques et perspectives en sociologie de la déviance », Sociétés contemporaines, 38, pp. 99-116.

Mossuz-Lavau J., 2005. - « Qui sont les clients ? » dans M.-É. Handman, J. Mossuz-Lavau (dirs.), La prostitution à Paris, Paris, Éditions de la Martinière, pp. 295-322.

O'Connell Davidson J., 2001. - « The sex tourist, the expatriate, his ex-wife and her "other" : the politics of loss, difference and desire », Sexualities, 4, 1, pp. 5-24.

Pheterson G., 2003. - «Grossesse et prostitution. Les femmes sous la tutelle de l'État », Raisons politiques, 11, pp. 97-116.

Prieur A., Taksdal A., 1993. - « Clients of prostitutes. Sick deviants or ordinary men ? A discussion of the male role concept and cultural changes in masculinity », NORA, 2, pp. 105-114.

Pryen S., 1999. - Stigmate et métier. Une approche sociologique de la prostitution de rue, Rennes, Presses Universitaires de Rennes.

- 2002. - «Prostitution de rue : le privé des femmes publiques », Ethnologie française, 1, pp. 11-18.

Redoutey E., 2005. - «Trottoirs et territoires, les lieux de la prostitution à Paris » dans M.-É. Handman, J. Mossuz-Lavau (dirs.), La prostitution à Paris, Paris, Éditions de la Martinière, pp. 39-89. 


\section{Revue française de sociologie}

Schauer C., 2003. - Kinder auf dem Strich. Bericht von der deutsch-tschechischen Grenze, Bad Honnef, UNICEF/ECPAT, Horlemann Verlag.

- 2006. - «Jeder holt sich, was er will. Sexuelle Ausbeutung von Frauen und Kindern in einer tschechischen Grenzregion », Osteuropa, 6, pp. 235-244.

Š́́dová L., 2004a. - «Organizace bulharské prostituce v České republice », Migrace online, Multikulturní centrum Praha, disponible sur : http://migraceonline.cz/clanky_f.shtml?x=210976 (25/06/2006).

- 2004b. - «Ukrajinská prostituce v České republice a způsob její organizace », Migrace online, Multikulturní centrum Praha, disponible sur: http://migraceonline.cz/clanky_f.shtmlx=214376 (25/06/2006).

Siegmund J., 2006. - «Die unbekannte Spezies. Ein Versuch, sich dem Phänomen Freier zu nähern », Osteuropa, 6, pp. 87-98.

Sykes G. M., Matza D., 1957. - «Techniques of neutralization : a theory of delinquency », American sociological review, 6, pp. 664-670.

Velten D., 1994. - Aspekte der sexuellen Sozialisation. Eine Analyse qualitativer Daten zu biographischen Entwicklungsmustern von Prostitutionskunden, Inauguraldissertation, Berlin, Freie Universität Berlin.

Watson P., 1993. - « Eastern Europe's silent revolution : gender », Sociology, 27, 3, pp. 471-487.

Welzer-Lang D., Barbosa O., Mathieu L., 1994. - Prostitution : les uns, les unes et les autres, Paris, Éditions Métailié. 